\title{
SGTPy: A Python code for calculating the interfacial properties of fluids based on the Square Gradient Theory using the SAFT-VR Mie equation of state
}

\author{
Andrés Mejía ${ }^{1, *}$, Erich A. Müller ${ }^{2}$, Gustavo Chaparro Maldonado ${ }^{1}$ \\ 1 Departamento de Ingeniería Química, Universidad de Concepción, Concepción 4030000, Chile. \\ 2 Department of Chemical Engineering, Imperial College London, London SW7 2AZ, United Kingdom.
}

\section{Walk-through: Description and Application of the SGTPy Package}

This document is devoted to describing the SGTPy package through selected step by step cases by using Jupyter notebooks. In general terms, the SGTPy package is divided into two main modules. The first one concerns the EoS model, while the second one the SGT calculations. This modularity facilitates the eventual replacement of the SAFT-VR-Mie EoS, for other versions of SAFT or other types of EoS. ${ }^{1}$ In the EoS module, the users must provide molecular parameters or employ the built-in optimization options to determine them. The second module solves the SGT for pure fluids and fluid mixtures. In this latter module, the influence parameter for pure fluids can be obtained by fitting to experimental data or entered as a user-supplied value. Similarly, for mixtures, the module allows the assignment of $\beta_{i j}\left(0 \leq \beta_{i j}<1\right)$ or it is fitting from data.

From a numerical perspective, the SGTPy package uses Python packages such as NumPy, ${ }^{2} \mathrm{SciPy},{ }^{3}$ and Cython. ${ }^{4}$ In order to reduce to amount to code the final user has to deal with, the package was coded in an object-oriented style. Previous to the use of SGTPy, it is necessary to download Python from its official website ${ }^{5}$ and the numerical packages from PyPI. ${ }^{6}$

\footnotetext{
* Corresponding author: amejia@udec.cl
} 


\section{Pure fluids on SGTPy}

To start using SGTPy is necessary to define the pure fluids with the function component, as illustrated below:

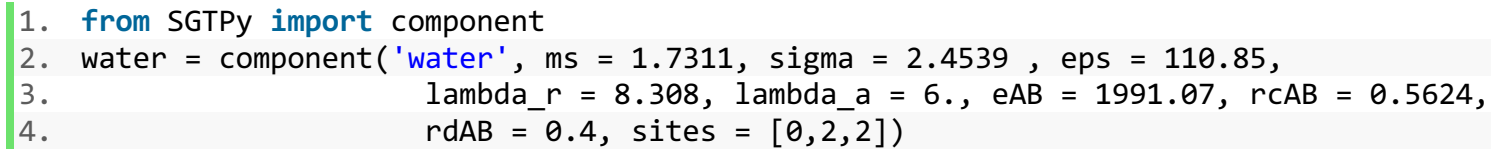

Notebook W1. Parameters definition for pure fluids on SAFT VR Mie EoS (e.g., water)

The component function creates a pure component and stores the molecular parameters for SAFTVR-Mie EoS. For the case of non-associating components, five parameters are necessary, namely: the segments number, $m_{s}$, the Mie energy potential, $\varepsilon$ in $1 / \mathrm{K}$ units, the Mie length scale, $\sigma$ in $\AA\left(10^{-}\right.$ ${ }^{10} \mathrm{~m}$ ) units; the repulsive and attractive exponents $\lambda_{r}$ and $\lambda_{a}$, respectively. For the case of pure selfassociating fluid, three extra parameters are needed: the association site energy, $\varepsilon^{A B}$ in $1 / \mathrm{K}$ units, the association range, $r_{c}{ }^{A B} / \sigma$, and the association scheme which is characterized by the list $[\mathrm{B}, \mathrm{P}$, $\mathrm{N}]{ }^{7,8}$ The equivalence between traditional association schemes and the $[\mathrm{B}, \mathrm{P}, \mathrm{N}]$ triplet are shown in Table W1.

Table W1. Association schemes in SGTPy.

\begin{tabular}{|c|c|c|}
\hline Association scheme & {$[\mathrm{B}, \mathrm{P}, \mathrm{N}]$} & Site Representation \\
\hline 1 & {$[0,0,1]$} & $-\frac{a}{1}$ \\
\hline $2 \mathrm{~B}$ & {$[0,1,1]$} & 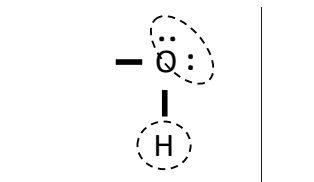 \\
\hline $2 \mathrm{C}$ & {$[1,0,1]$} & $\begin{array}{l}\mathbf{I} \\
\mathrm{H} \\
\mathbf{1}\end{array}$ \\
\hline
\end{tabular}




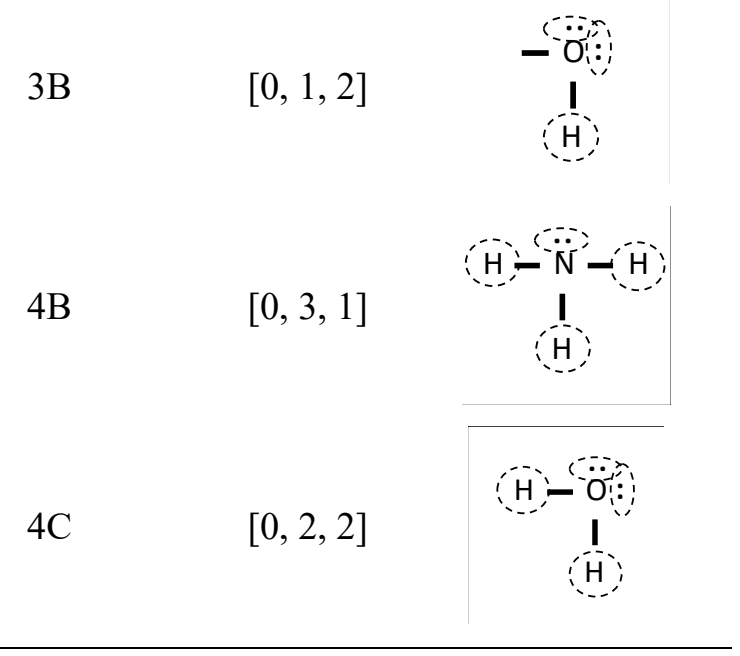

Finally, the polar contribution requires the definition of a dipolar moment, $\mu$ in Debye units, and the number of polar sites, $n_{p}$. If any of the molecular parameters is not specified, SGTPy will take a default value which implies that the association or polar contribution might not be considered. In the cases when the SAFT-VR-Mie parameters are not available or the user decides to optimize their values, SGTPy uses the experimental values provided by the user, and the following notebook can be used to obtain their numerical values.

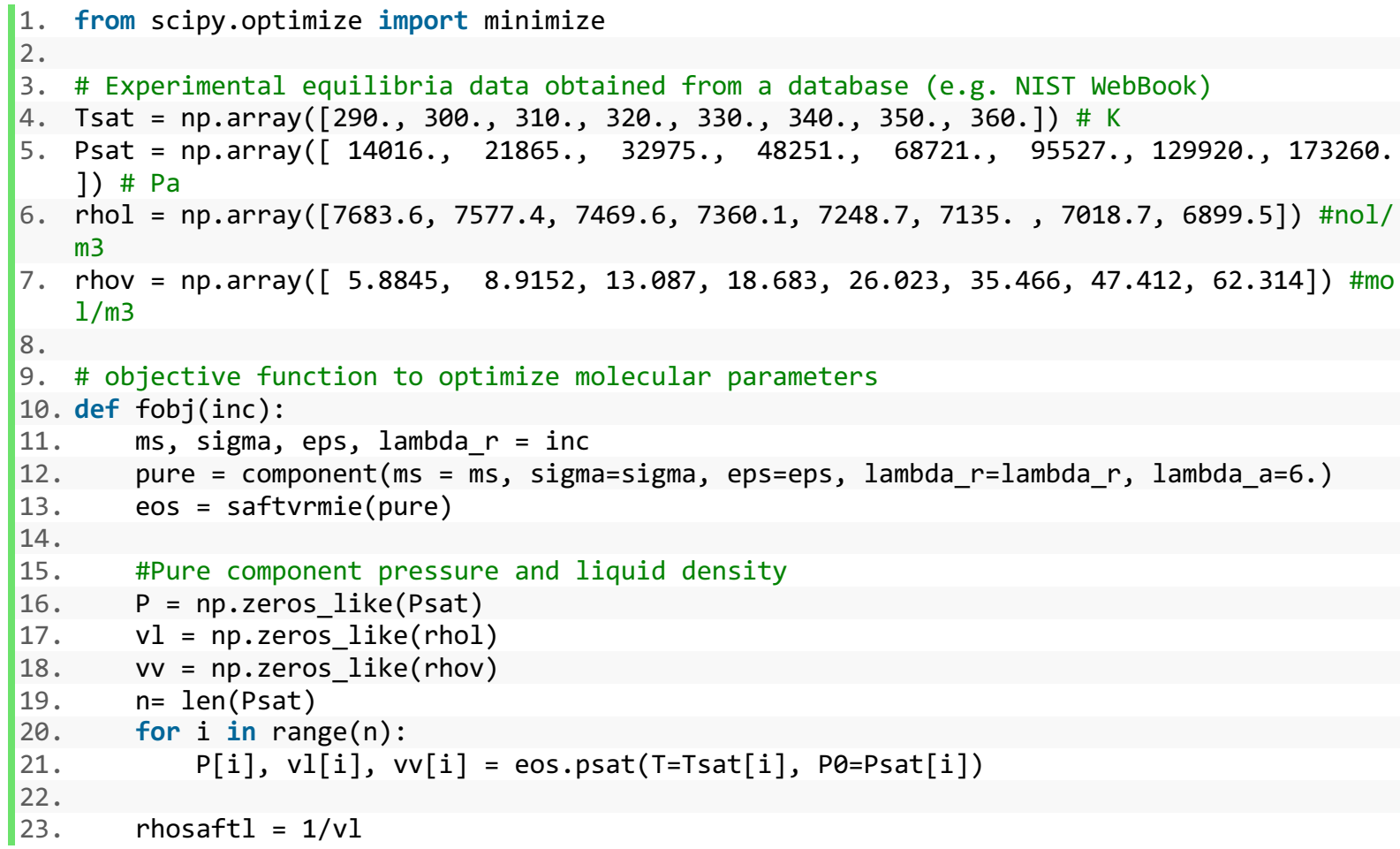




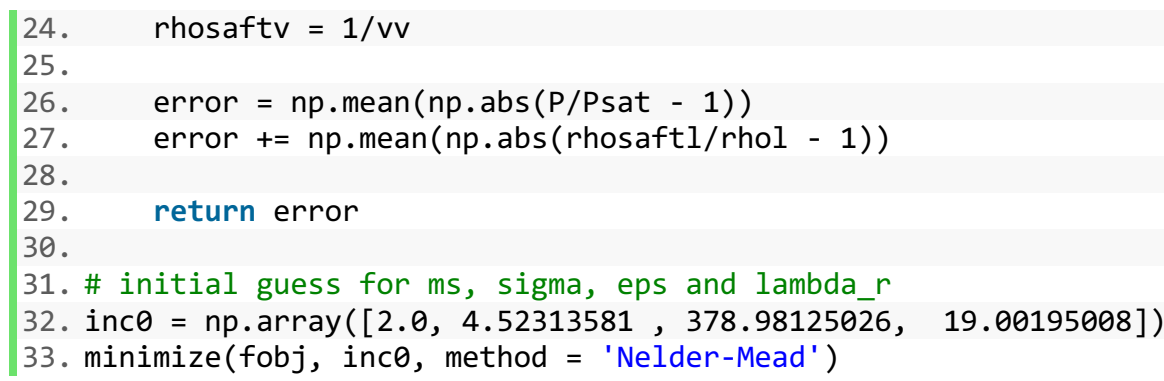

Notebook W2. Optimization of the SAFT VR Mie parameters for pure fluids (e.g., n-hexane).

In Notebook W2, the 'Nelder-Mead' method is used to optimize four parameters ( $m_{s}, \varepsilon, \sigma$ and $\left.\lambda_{r}\right)$, however, when the optimization involves more parameters (i.e., associating or polar fluids) other optimization methods could be more suitable. It is advised the SciPy's minimize documentation ${ }^{3}$ to choose the best algorithm for each problem.

Nevertheless, for the case of association parameters, they can be obtained from the original sources or from Cripwell et al., ${ }^{9}$ and Dufal et al., ${ }^{10}$ who reported a significant group of parameters for several types of fluids. Otherwise, it is advisable to follow Cripwell et al. work for further guidelines to obtain new parameters. In this version of SGTPy, this latter functionality has not been implemented yet.

Table W2 summarizes some selected numerical examples of the molecular parameters for pure fluids obtained and used in SGTPy for the numerical examples in this work.

Table W2. Selected SAFT-VR-Mie and SGT parameters for pure fluid. ${ }^{1,2,3}$

\begin{tabular}{lcccccccccc}
\hline \multicolumn{1}{c}{ Fluid } & $m_{s}$ & $\sigma / \AA$ & $\varepsilon / k_{B} /$ & $\lambda_{r}$ & $\varepsilon^{A B} / k_{B} /$ & $r_{c}^{A B} / \sigma$ & {$[\mathrm{B}, \mathrm{P}, \mathrm{N}]$} & $\mu / \mathrm{D}$ & $n_{p}$ & $\begin{array}{c}\boldsymbol{c}_{i \boldsymbol{i}} \times \mathbf{1 0}^{\mathbf{2 0} / \mathbf{J}} \\
\mathbf{m}^{5} \mathbf{~ m o l}^{-2}\end{array}$ \\
\hline n-Butanol $^{\mathrm{d}}$ & 1.965 & 4.108 & 277.89 & 10.668 & 3300 & 0.2615 & {$[1,0,1]$} & 1.66 & 1.45 & 15.018 \\
CPME $^{\mathrm{a},{ }^{*}}$ & 2.325 & 4.136 & 343.91 & 14.155 & -- & -- & {$[0,0,1]$} & 1.27 & 1.919 & 35.213 \\
Dodecane $^{\mathrm{c}}$ & 4.000 & 4.351 & 378.56 & 18.410 & - & -- & -- & -- & -- & 127.21 \\
Ethanol $^{\mathrm{d}}$ & 1.772 & 3.559 & 224.50 & 11.319 & 3018.05 & 0.3547 & {$[1,0,1]$} & -- & -- & 5.3141 \\
Hexane $^{\mathrm{b}}$ & 1.967 & 4.547 & 377.60 & 18.410 & -- & -- & -- & -- & -- & 35.815 \\
Methane $^{\mathrm{c}}$ & 1.000 & 3.752 & 170.75 & 16.390 & -- & -- & -- & -- & -- & 1.9207 \\
MTBE $^{\mathrm{a},{ }^{*}}$ & 2.178 & 4.191 & 306.52 & 14.741 & -- & -- & {$[0,0,1]$} & 1.36 & 2.951 & 35.779 \\
Water $^{\mathrm{d}}$ & 1.731 & 2.454 & 110.85 & 8.308 & 1991.07 & 0.5624 & {$[0,2,2]$} & & & 1.5372 \\
\hline
\end{tabular}


* CPME: Cyclopentyl methyl ether and MTBE: Methyl tert-butyl ether.

${ }^{1}$ the values of $\lambda_{a}$ and $r_{d}{ }^{A B} / \sigma$ are fixed to 6 and 0.4 , respectively. ${ }^{2}$ The numerical values of SAFT-VR-Mie parameters are obtained from the experimental data: ${ }^{\mathrm{a}}$ DECHEMA database, ${ }^{11 \mathrm{~b}}$ TDE-NIST database, ${ }^{12}$ and taken from : ${ }^{\mathrm{c}}$ Mejía et al., ${ }^{13 \mathrm{~d}}$ Cripwell et al., ${ }^{9}$ the influence parameters have been optimized from experimental data for CPME from Mejia and Cartes; ${ }^{14}$ for ethanol, hexane, water, 1-butanol, and MTBE from TDE-NIST database, ${ }^{12}$ and Equation (11) is used for methane and dodecane.

Now that the component is defined (e.g., water), it can be modeled with SAFT-VR-Mie EoS.

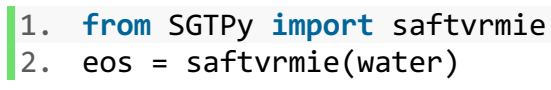

Notebook W3. Transfer the SAFT VR Mie parameters for pure fluid to the EoS module.

The eos object includes all the necessary functions to evaluate density, pressure, chemical potential, and fugacity coefficients. All the included methods use SI units: temperature in Kelvin, pressure in $\mathrm{Pa}$, and density in $\mathrm{mol} / \mathrm{m}^{3}$. The eos.psat method computes the saturation pressure at a given temperature and returns the saturation pressure and the volume roots of the liquid and vapor phase, if no initial guess for the volumes are given they are initiated by the Topliss's method. ${ }^{15}$ As an illustration, the following notebook calculates the phase equilibrium for pure fluids.

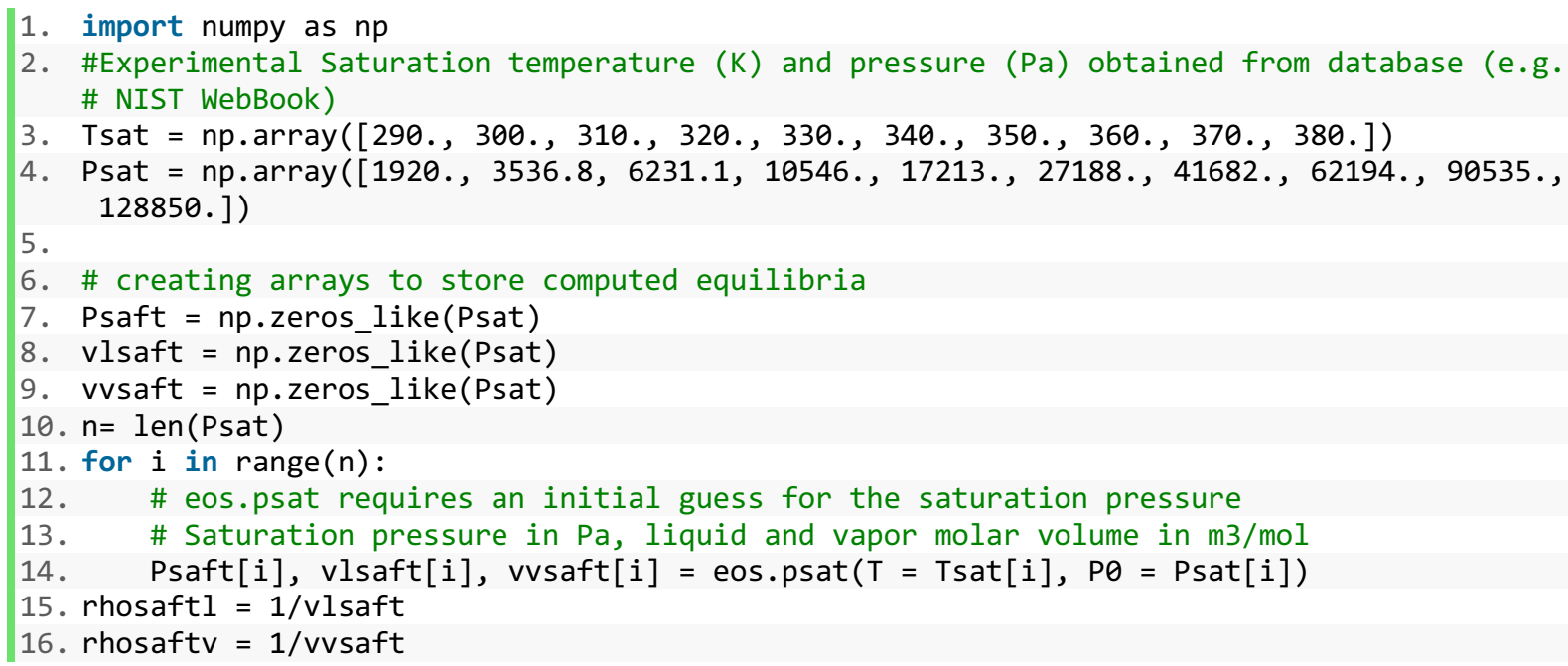

Notebook W4. Phase equilibrium calculations for pure fluids using SAFT VR Mie EoS (e.g., water). 
In order to calculate the interfacial properties for a fluid from SGTPy, it is necessary to include the numerical value of the influence parameter, $c_{i i}$ in $\mathrm{J} \mathrm{m}^{5} \mathrm{~mol}^{-2}$ units in component, as it is illustrated below:

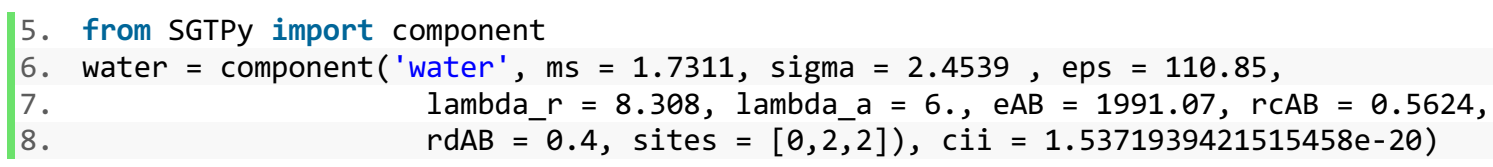

Notebook W5. Definition of pure fluids parameters for phase equilibrium and interfacial properties (e.g., water).

The numerical value of $c_{i i}$ can be obtained for the literature or calculated from Equation (11) for the case of pure non-associating CG Mie chains, otherwise it is fixed from Equation (10) and experimental values of the surface tension. The latter procedure is illustrated in the following codeblock.

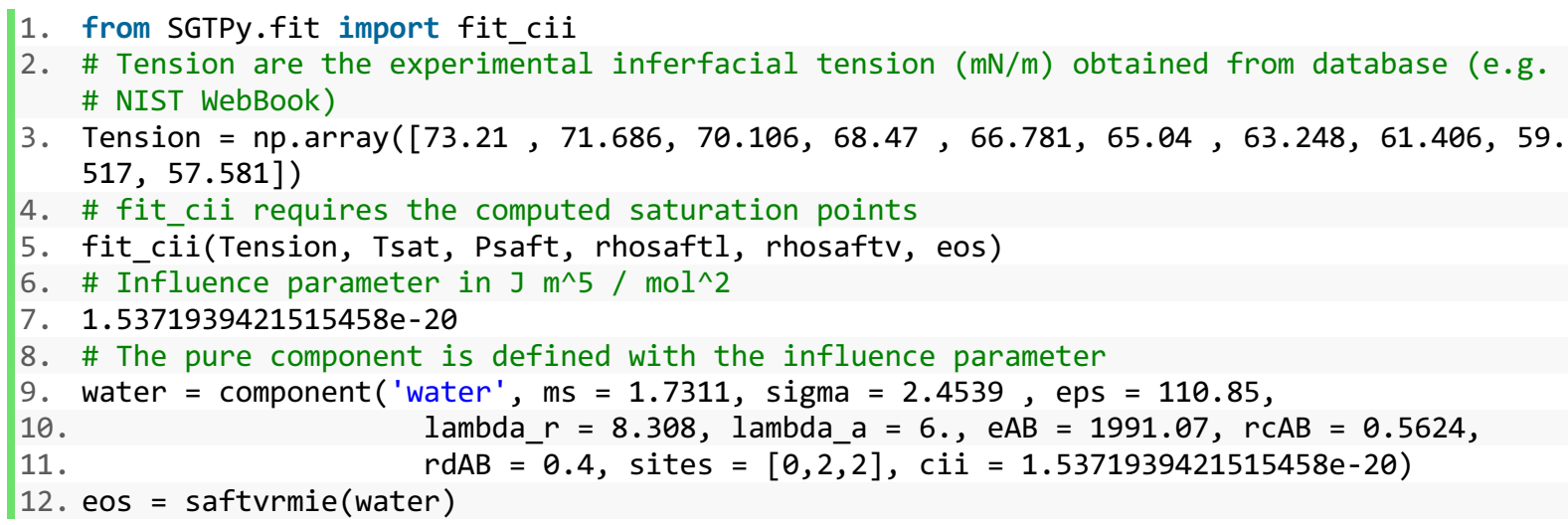

Notebook W6. Optimization of the influence parameter for pure fluids using experimental data of surface tension

$$
\text { (e.g., water). }
$$

Table W2 summarizes the numerical values of $c_{i i}$ for the pure fluids using in this work.

Now the eos object is capable of computing interfacial tension with the sgt_pure function. The procedure is straightforward as it is shown below.

1. from SGTPy.sgt import sgt_pure

2. \# array to store computed interfacial tension

3. tensaft $=$ np.zeros_like(Tension)

4. for $i$ in range( $n)$ :

5. \# interfacial tension result is outputted in $\mathrm{mN} / \mathrm{m}$

6. tensaft $[i]=$ sgt_pure(rhosaftv[i], rhosaftl[i], Tsat[i], Psaft[i], eos)

Notebook W7. Surface tension for pure fluids from SGT + SAFT VR Mie EoS (e.g., water) 
The results are compared against experimental data in the following Figure.

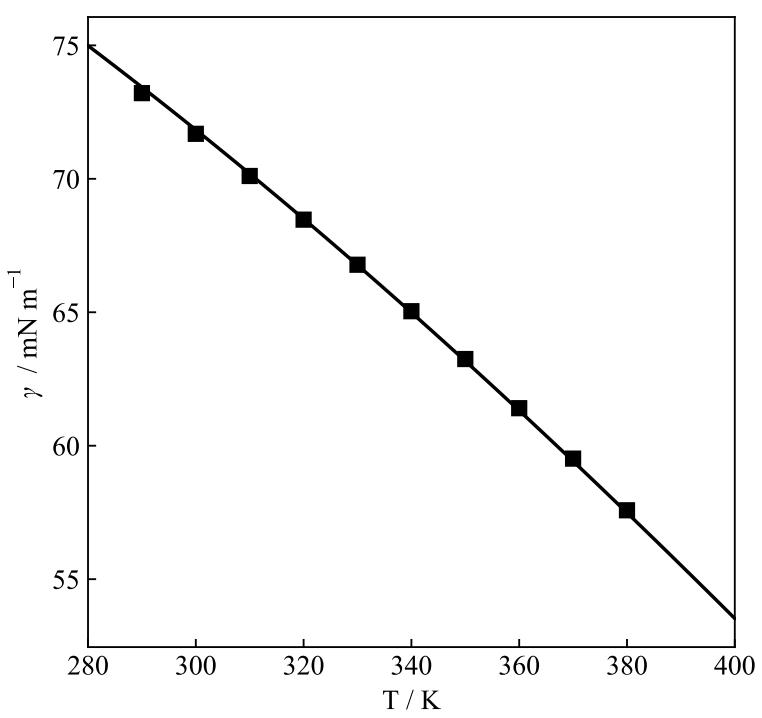

Figure W1. Interfacial tension, $\gamma$, as a function of temperature, $T$, for water. - , Predictions based on

$$
\mathrm{SGT} ; \boldsymbol{\square}, \text { TDE- NIST. }^{12}
$$

\section{Fluid Mixtures on SGTPy Package}

Binary mixture in vapor-liquid equilibria (VLE) with $\beta_{i j}=0$.

For the case of mixtures, the first step is to create the mixture. For example, in a binary mixture, the function mixture takes two pure components and creates a mixture with them, defining the interaction parameters (or obtaining them from the fitting to experimental data). For illustration, the following notebook considers the optimization of the $k_{i j}$ parameter for the case of methane (1) + dodecane (2) mixture.

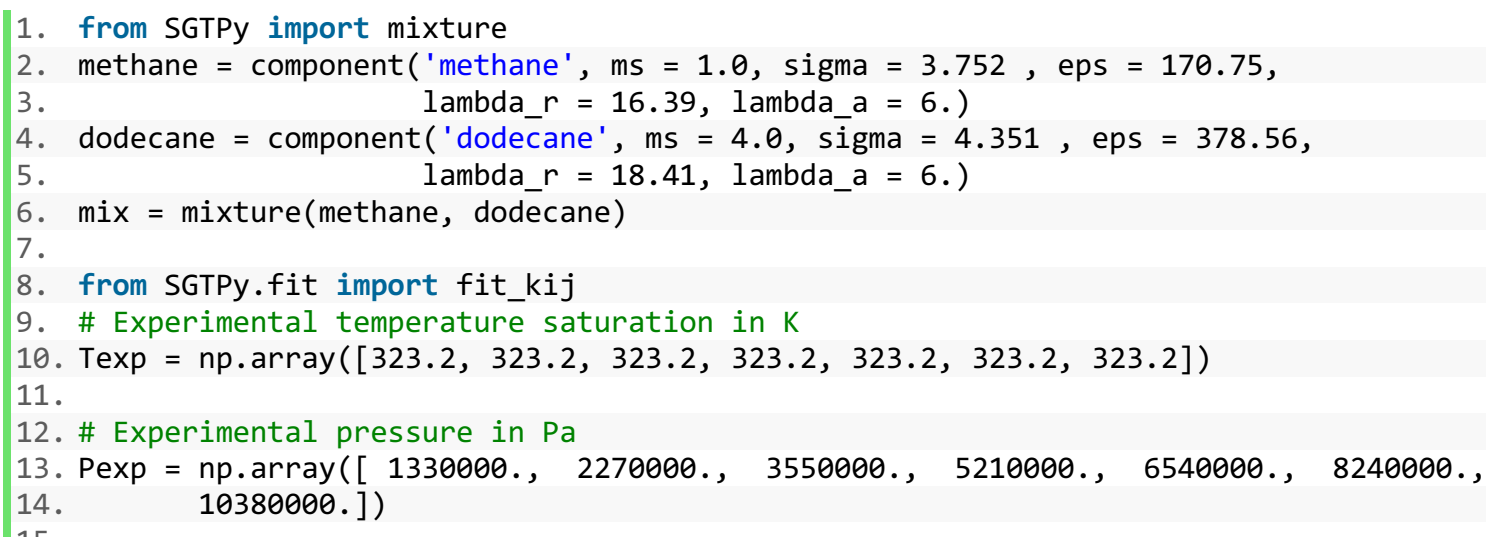




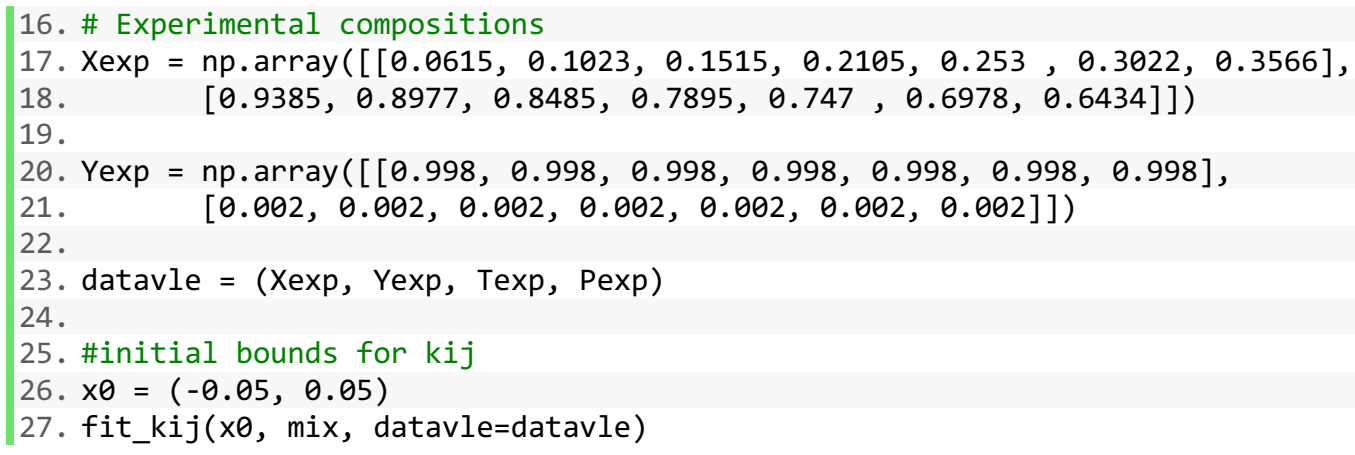

Notebook W8. Optimization of the binary parameter $k_{i j}$ for methane + dodecane mixture.

From the previous notebook, $k_{i j}=-0.02199$, which is included in the notebook for the mixture, as it is illustrated in the Notebook W9.

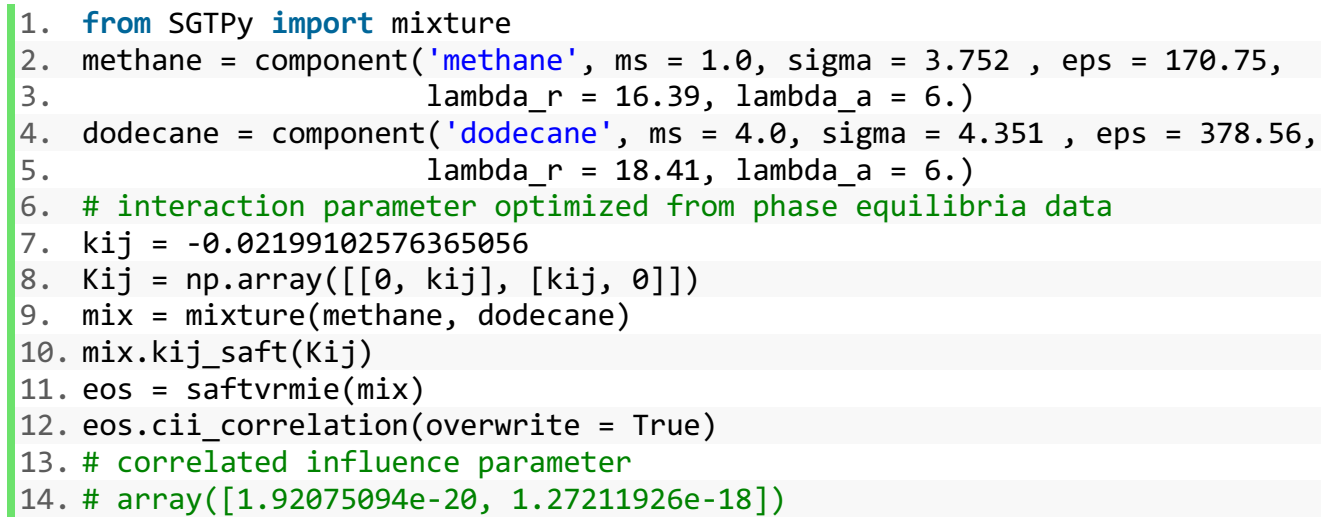

Notebook W9. Binary mixture creations for SAFT VR Mie EoS (e.g., methane + dodecane)

In the previous code-block a mixture of methane (1) + dodecane (2) is created, then an interaction parameter $\left(k_{i j}\right)$ fitted from experimental equilibria data ${ }^{11}$ is set through the eos.kij_saft method. Finally, the influence parameters $\left(c_{i i}\right.$ and $c_{j j}$ ) are correlated from the molecular parameters (see Equation (11) of main text). ${ }^{16}$

Table W3 summarizes the interaction parameters used for the phase equilibria of the mixtures explored in this work and their respective statistics. Specific details of each mixture are described later. 
Table W3. Interaction parameters for fluid mixtures and statistics ${ }^{\mathrm{a}, \mathrm{b}}$

\begin{tabular}{|c|c|c|c|c|c|c|c|c|}
\hline$i$ & $j$ & $k_{i j}$ & $\boldsymbol{r}_{c}^{A B i j}(\AA)$ & $\% \Delta P^{a}$ & $\% \Delta y^{b}$ & $\% \Delta x^{b}$ & $\% \Delta w^{b}$ & Ref. \\
\hline Methane & Dodecane & -0.0219 & -- & 1.21 & -- & -- & -- & 17 \\
\hline Ethanol & Hexane & 0.0118 & -- & 0.88 & 0.88 & -- & -- & 18 \\
\hline Ethanol & CPME & 0.0101 & 2.2315 & 0.91 & 0.73 & -- & -- & 19 \\
\hline CPME & Hexane & 0.0008 & -- & 0.82 & 0.43 & -- & -- & 20 \\
\hline MTBE & 1-Butanol & -0.0029 & 1.9098 & 0.63 & 0.81 & -- & -- & 21 \\
\hline Water & 1-Butanol & -0.0073 & -- & -- & -- & 2.25 & 1.71 & 22,23 \\
\hline Water & MTBE & -0.0733 & 2.8436 & -- & -- & 1.57 & 0.32 & $24,25,26$ \\
\hline
\end{tabular}

${ }^{\mathrm{a}} l_{i j}=-0.0073$ for water +1 -butanol mixture and $l_{i j}=0$ for the other mixtures; ${ }^{\mathrm{b}}$ statistical deviations: $\% \Delta \mathrm{P}=(100 / \mathrm{n}) \sum_{\mathrm{i}=1}^{\mathrm{n}} \mid \mathrm{P}_{\mathrm{i}}^{\text {cal }}-\mathrm{P}_{\mathrm{i}}^{\text {exp }} / / \mathrm{P}_{\mathrm{i}}^{\text {exp }}$ and $\% \Delta \vartheta=(100 / \mathrm{n}) \sum_{\mathrm{i}=1}^{\mathrm{n}}\left|\vartheta_{\mathrm{i}}^{\text {cal }}-\vartheta_{\mathrm{i}}^{\text {exp }}\right|$, with $\vartheta_{i}: \mathrm{x}$, w or $\mathrm{y}$.

In SGTPy, the eos object can be simultaneously used to compute phase equilibria and to predict the interfacial tensions with SGT.

The Notebook W10 illustrates the calculation of the vapor-liquid equilibria (VLE) and interfacial tension for methane (1) + dodecane (2) at $344.15 \mathrm{~K}$ and $10 \mathrm{MPa}$. In this case, the VLE is computed using the flash calculation. The function flash requires an initial guess for the phase composition and the type of equilibria, 'LV' for liquid-vapor. Then, the density vectors are computed with the eos.density function. Finally, the assigned value of $\beta_{12}$ is equal to zero, and the interfacial tension is computed with sgt_mix_beta $\theta$ function using the component reference method.

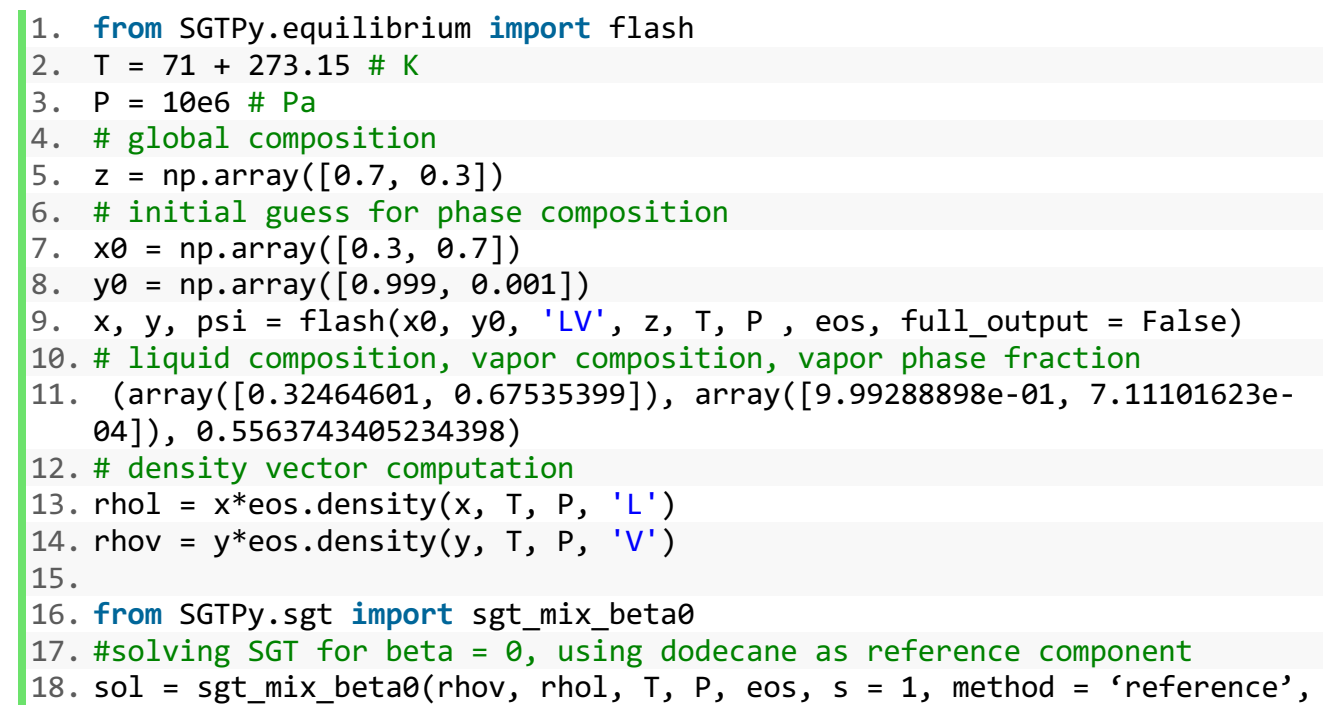


19. full_output = True)

20. \# interfacial tension $=\overline{1} 1.668198746517382 \mathrm{mN} / \mathrm{m}$

Notebook W10. Vapor - liquid equilibrium and interfacial tension for methane + dodecane at $344.15 \mathrm{~K}$ and $10 \mathrm{MPa}$.

In the Notebook W10, the full_output option allows accessing interfacial tension, density path, and grand thermodynamic potential. This procedure can be repeated over a range of pressure or temperature. Figure W2 illustrates the concentration profiles along the interfacial region or in the concentration space projection $\left(\rho_{i}-z\right)$ for methane $(1)+$ dodecane (2) at $344.15 \mathrm{~K}$
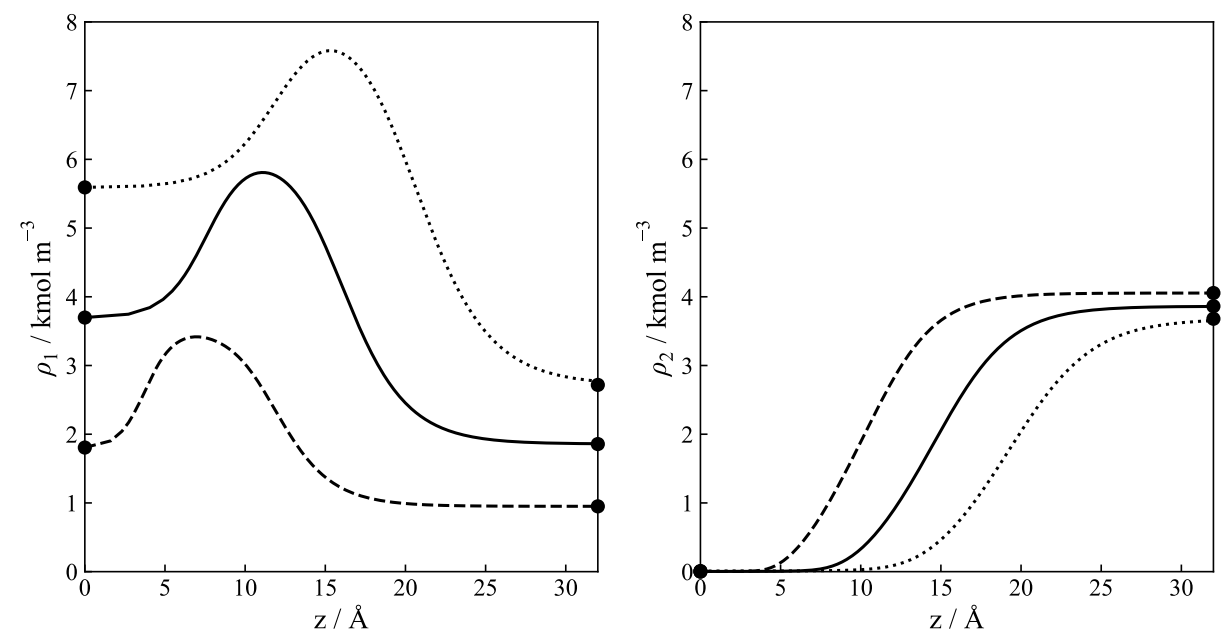

Figure W2. Interfacial concentration profile in $\rho_{i}-z$ projection for methane (1) + dodecane (2) at 344.15 K. SGT predictions at --, $5 \mathrm{MPa} ;-, 10 \mathrm{MPa} ; \cdots, 15 \mathrm{MPa}$. •, bulk phase equilibrium.

From Figure W2 it can be seen that there is a strong methane interfacial activity which implies accumulation of this component in the interface. This surface activity increases as the pressure increases. Figure W3 illustrates the prediction of bulk densities and the variation of the interfacial tension with the pressure for the methane (1) + dodecane (2) at $344.15 \mathrm{~K}$ and the corresponding comparison to densimetry and pendant drop tensiometry data. ${ }^{27}$ 

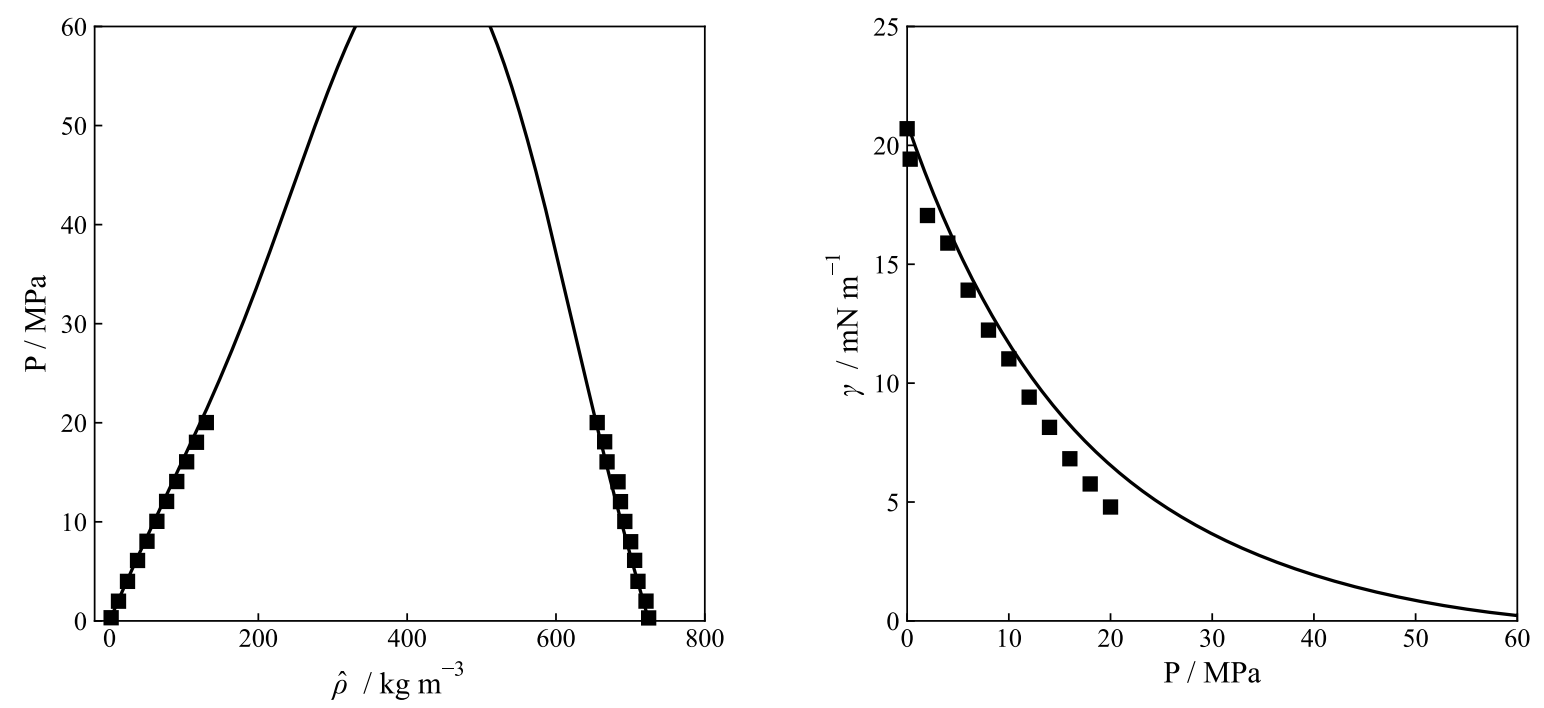

Figure W3. Bulk density and Interfacial tension for methane (1) + dodecane (2) at 344.15 K. - , SGT predictions; $\mathbf{\square}$, Cartes $^{27}$

There is a good agreement between experimentation and theory, within an absolute error of 5\% in vapor density, $0.6 \%$ in liquid density and $5.5 \%$ in interfacial tension. This predictive methodology is suitable for mixtures of chain non-associating components, it uses correlated molecular parameters and influence parameters and relies only on one fitted interaction parameter $\left(k_{i j}\right)$. (see Refs. 28, 29, 30 for other examples with $\mathrm{CO}_{2}+$ hydrocarbons).

\section{Binary mixture in vapor-liquid equilibria $(V L E)$ with $\beta_{i j} \neq 0$}

In lieu of experimental data, one can predict the interfacial behavior of hydrocarbon mixtures using a geometric mean rule for the cross-influence parameter with $\beta_{i j}=0$, as is illustrated in the previous example. However, other mixtures that exhibit some degree of non-ideality, require that $\beta_{i j}$ is adjusted. SGTPy has a provision to perform this minimization using the fit_beta function. The mixture of hexane (1) + ethanol (2) will be used to exemplify this procedure. The first step is to create the pure components and set the interaction parameter fitted to experimental data, ${ }^{18}$ as was illustrated in Notebook W8.

1. \#creating pure components

2. ethanol $=$ component $($ 'ethanol2C', $\mathrm{ms}=1.7728$, sigma $=3.5592$, eps $=224.50$, 


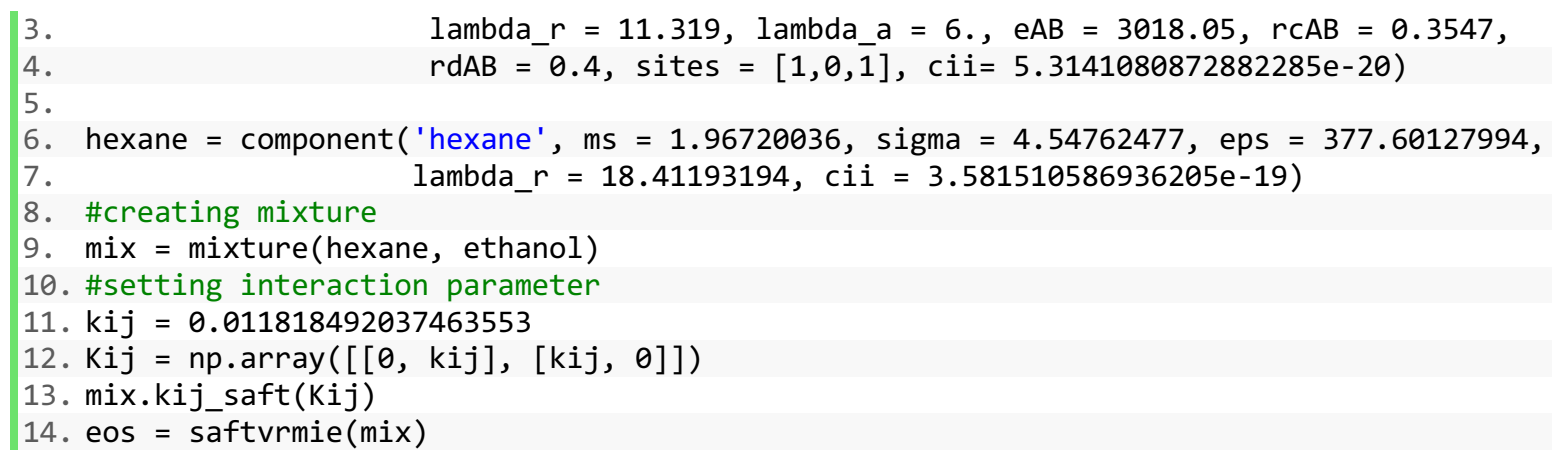

Notebook W11. Initial setup for the hexane (1) + ethanol (2) mixture.

Once the model has been set up, it can be used to compute the phase equilibrium at the experimental conditions. As it is illustrated in Notebook W12, initially the bubble points at a given temperature are computed, then the density vector of each phase is computed. The bubblePy function requires an initial guess for the vapor composition and the equilibrium pressure.

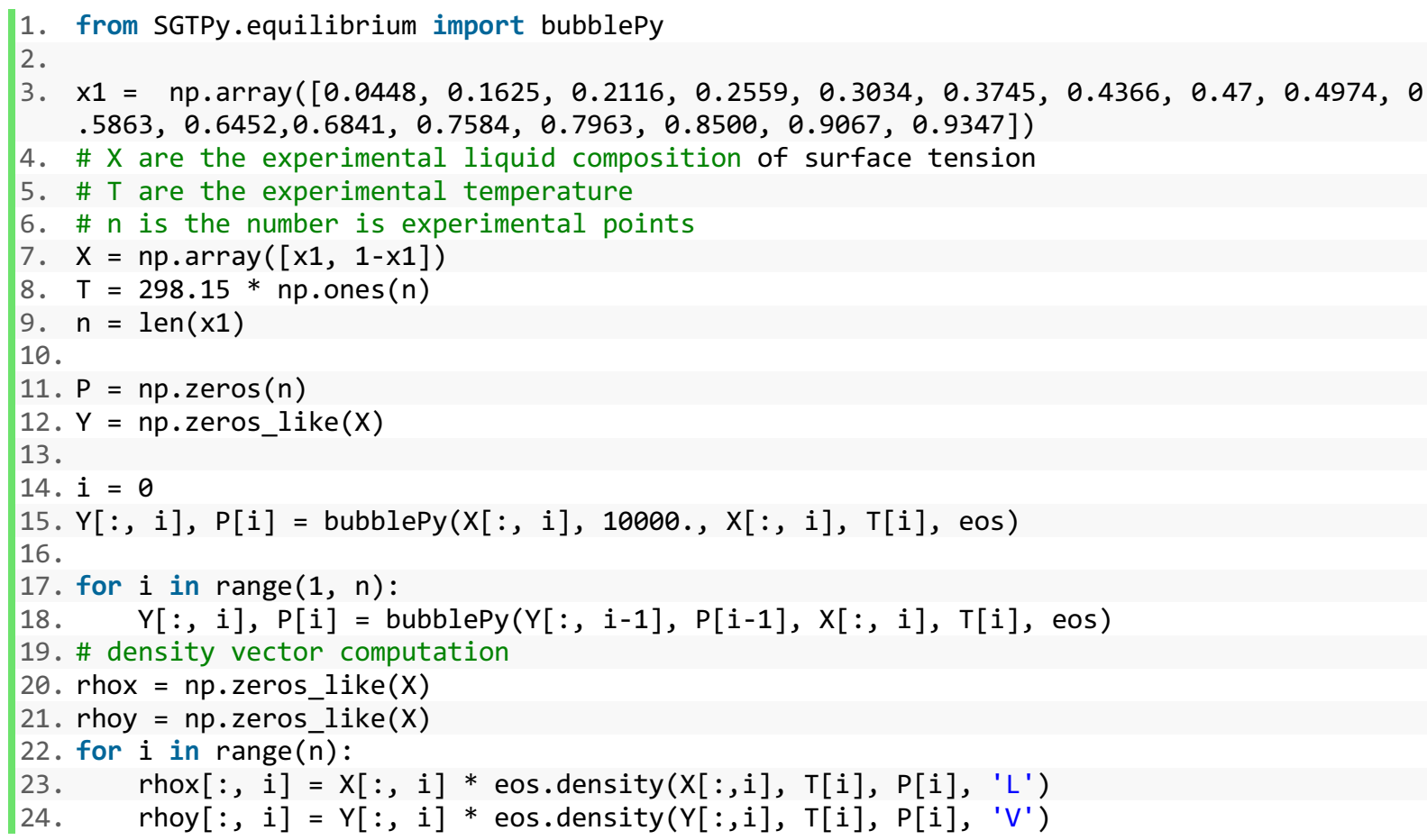

Notebook W12. Vapor - liquid equilibrium for hexane (1) + ethanol (2) mixture at $298.15 \mathrm{~K}$ 
Once the equilibrium information is calculated, the $\beta_{i j}$ correction can be optimized with the fit_beta function. This function is built on top SciPy's minimize function and requires bounds for this parameter. This procedure is illustrated in the following notebook.

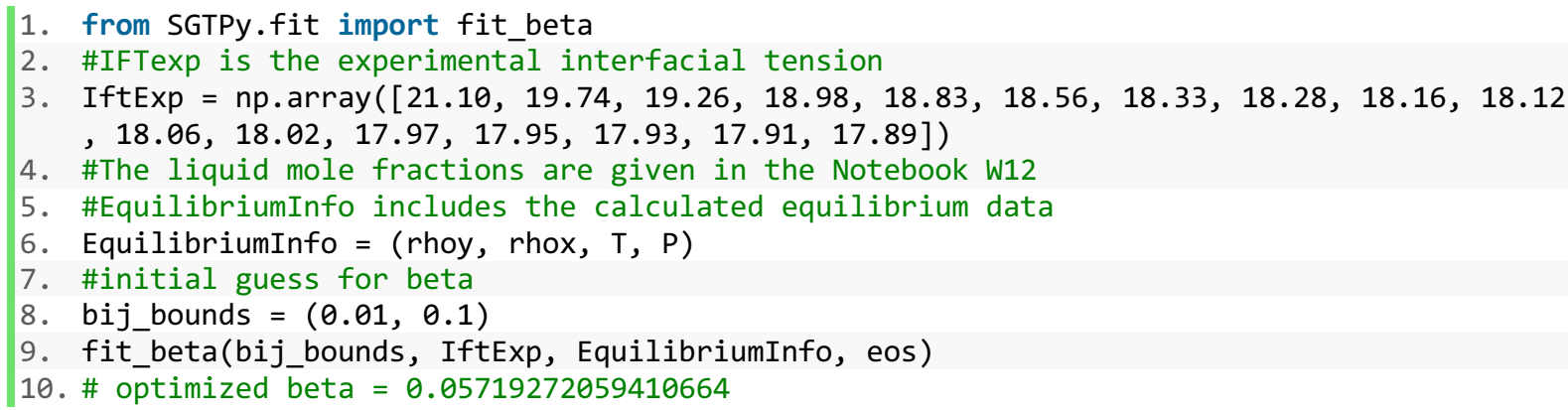

Notebook W13. Optimization of the symmetric cross interfacial tension parameter $(\beta)$ for hexane (1) + ethanol (2) mixture at $298.15 \mathrm{~K}$.

For this mixture, this procedure leads to an optimum of $\beta_{i j}=0.057$. As an example, the interfacial behavior is computed for this mixture at $x_{1}=0.3$ and $298.15 \mathrm{~K}$. Initially, the bubble point is computed with the bubblePy function, and the full_output option allows to obtain all the computed information needed such as the mole fraction vector, the volume of the phases and the association non-bonded sites fractions. Next, SGT will be applied for $\beta_{i j}=0$ using Liang's path function $^{31}$ and for $\beta_{i j}=0.057$ the BVP is solved by orthogonal colocation. ${ }^{32}$ The latter calculations are illustrated in the following code-block.

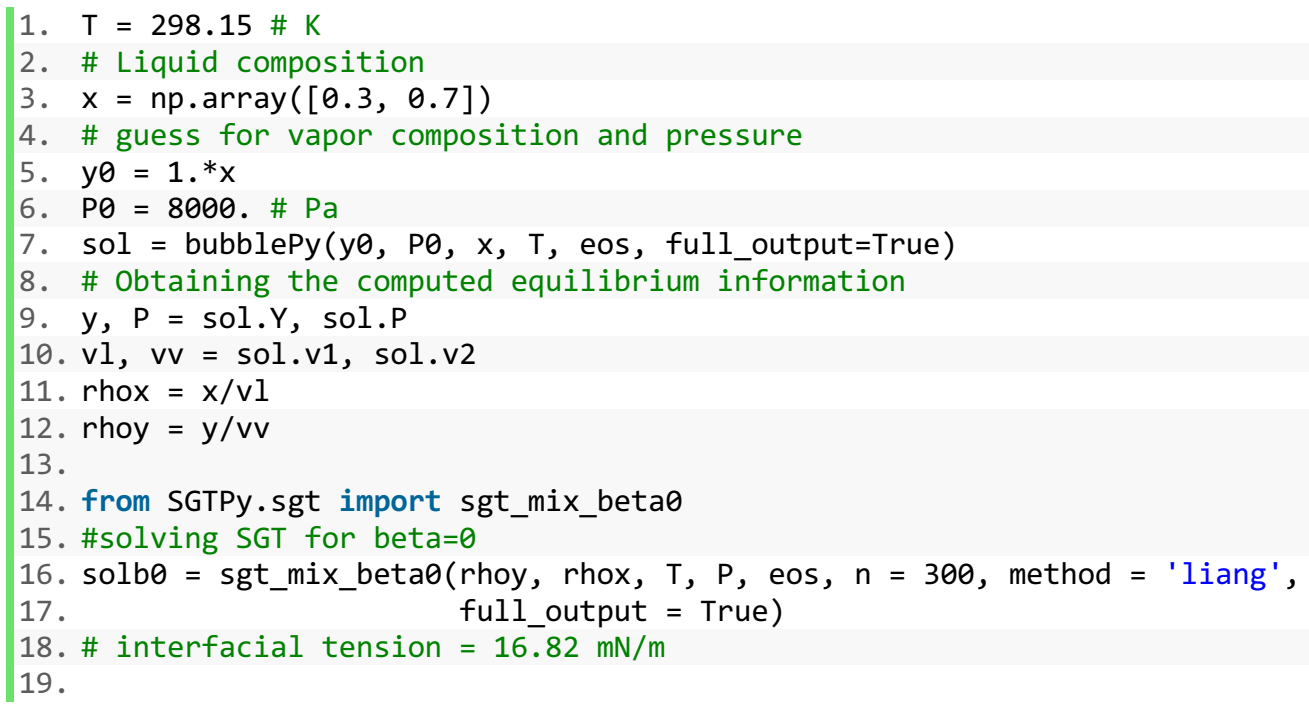




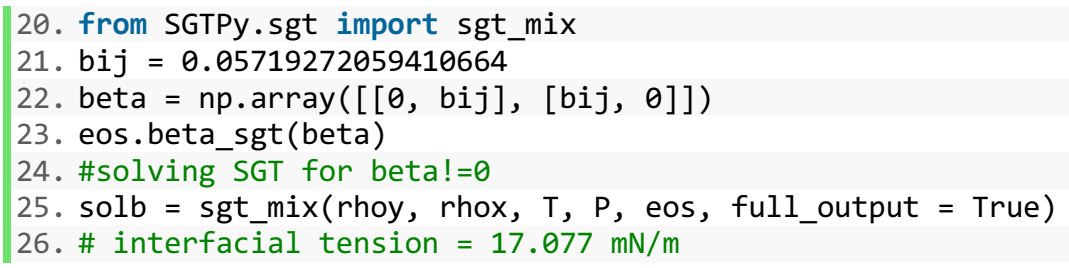

Notebook W14. Calculation of the Interfacial tension for mixtures using $\beta_{i j}=0$ and $\beta_{i j}=0.057$ for hexane (1) + ethanol (2) mixture at $x_{l}=0.3$ and $298.15 \mathrm{~K}$.

The predicted interfacial tension $\left(\beta_{i j}=0\right)$ and the calculated interfacial tension $\left(\beta_{i j} \neq 0\right)$ over the entire composition range are shown in Figure W4, where it is possible to observe that the correction for the cross-influence parameter slightly improves the prediction of the interfacial tension of the mixture, reducing the deviation from $7.36 \%$ to $6.24 \%$.

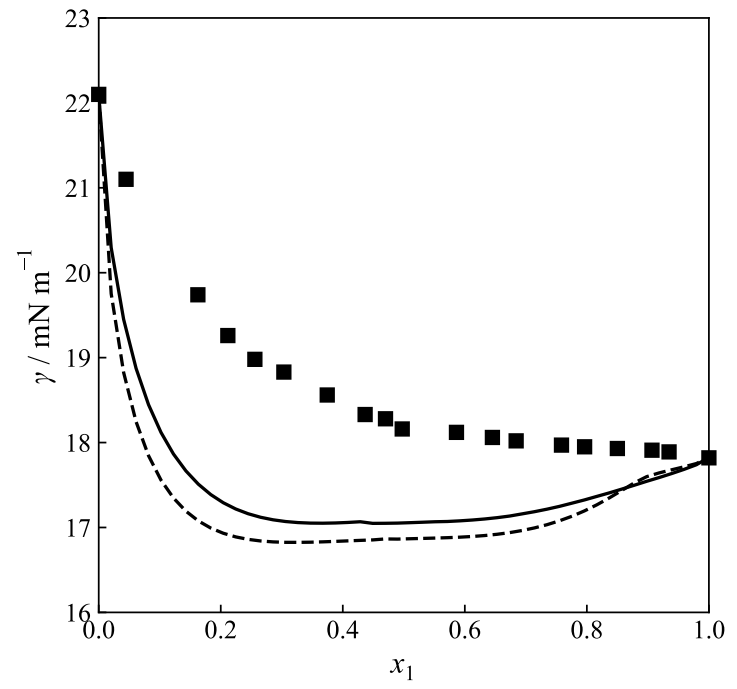

Figure W4. Interfacial tension $(\gamma)$ as a function of the liquid mole fraction $\left(x_{1}\right)$ for hexane $(1)+$ ethanol (2) mixture at $298.15 \mathrm{~K}$. SGT results: - -,$\beta_{i j}=0 ;-, \beta_{i j}=0.057$. $\mathbf{\square}$, Jiménez et al., ${ }^{33}$

However, the effect of an optimized $\beta_{i j}$ is more notorious on the density space projection, as it is shown on Figure W5. 

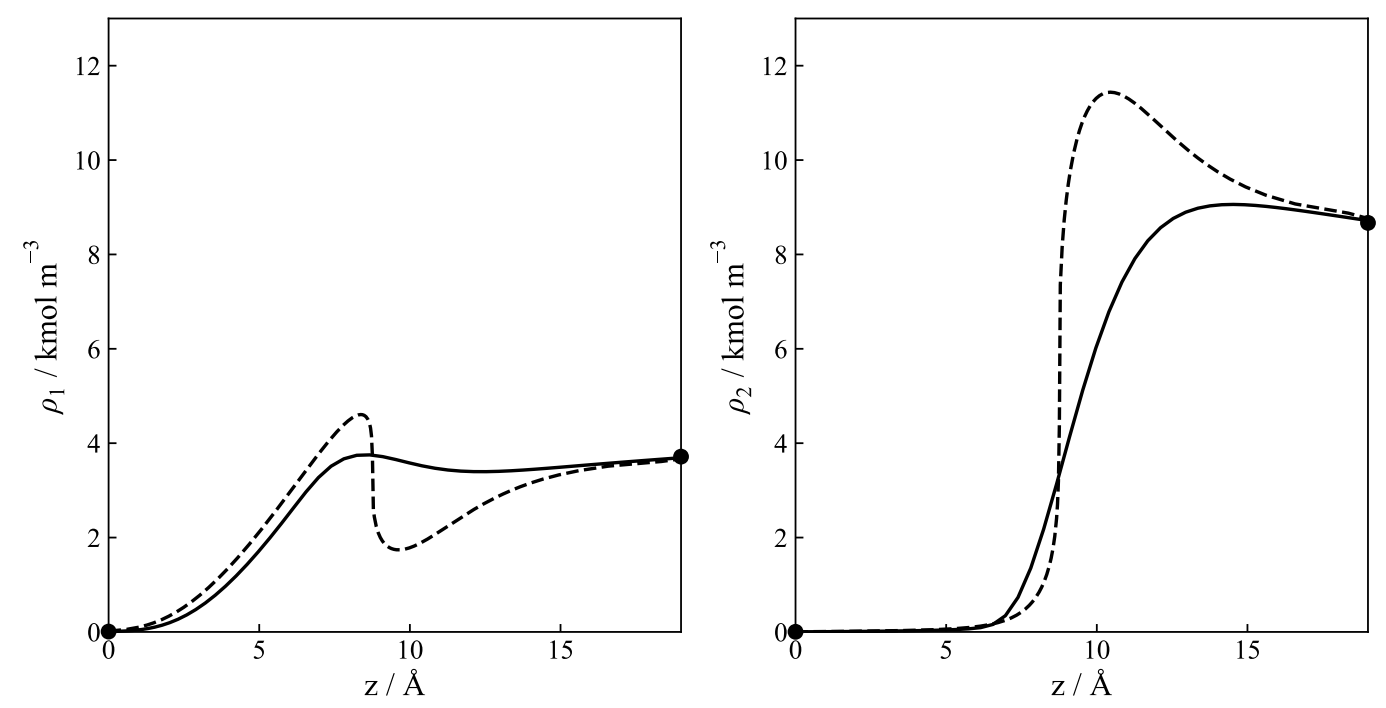

Figure W5. Interfacial concentration profile in $\rho_{i}-z$ projection for hexane (1) + ethanol (2) mixture at $x_{1}=0.3$ and $298.15 \mathrm{~K}$. SGT results: - -,$- \beta_{i j}=0 ;-, \beta_{i j}=0.057 ; \bullet$, bulk phase equilibrium.

From Figure W5, it is noted that the concentration profile with $\beta_{i j}=0$ shows several stationary points for hexane, which implies that the species exhibits simultaneously desorption and absorption. This behavior is inconsistent and unexpected but is corrected when an appropriate cross-influence parameter is applied: the true interfacial tension calculated from SGT + SAFT VR Mie corresponds to that with $\beta_{i j}=0.057$. Notwithstanding, it is evident that better models might be needed to calculate the interfacial tension for this type of mixtures. This lack of accuracy has been also noted by Cornelisse ${ }^{34}$ for other n-alkane + ethanol mixtures, using different EoSs, and also Liang et al., ${ }^{31}$ describes in depth the numerical problems for this type of mixtures.

The methodology allows optimization of interaction parameters for pure fluids $\left(c_{i i}, c_{j j}\right)$ and the cross-influence parameter for binary mixtures $\left(\beta_{i j}\right)$, implying a fully predictive approach for mixtures of three or more components. As a third illustration, the ternary mixture of hexane (1) + ethanol (2) + CPME (3) is selected. In this case, the ether is modeled as a polar molecule that can associate with ethanol. Previous to building a ternary mixture, it is necessary to obtain all binary interaction parameters. 


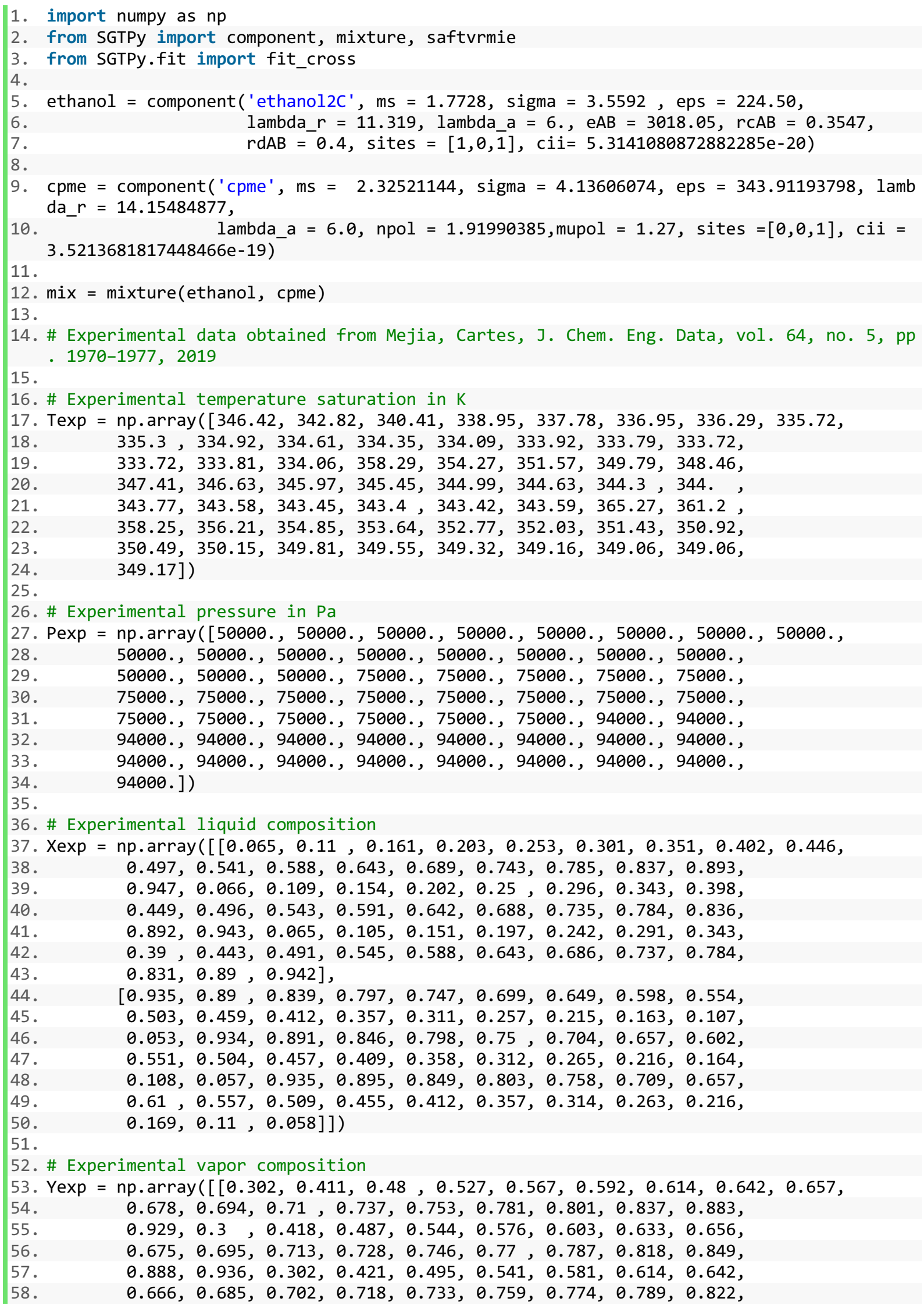




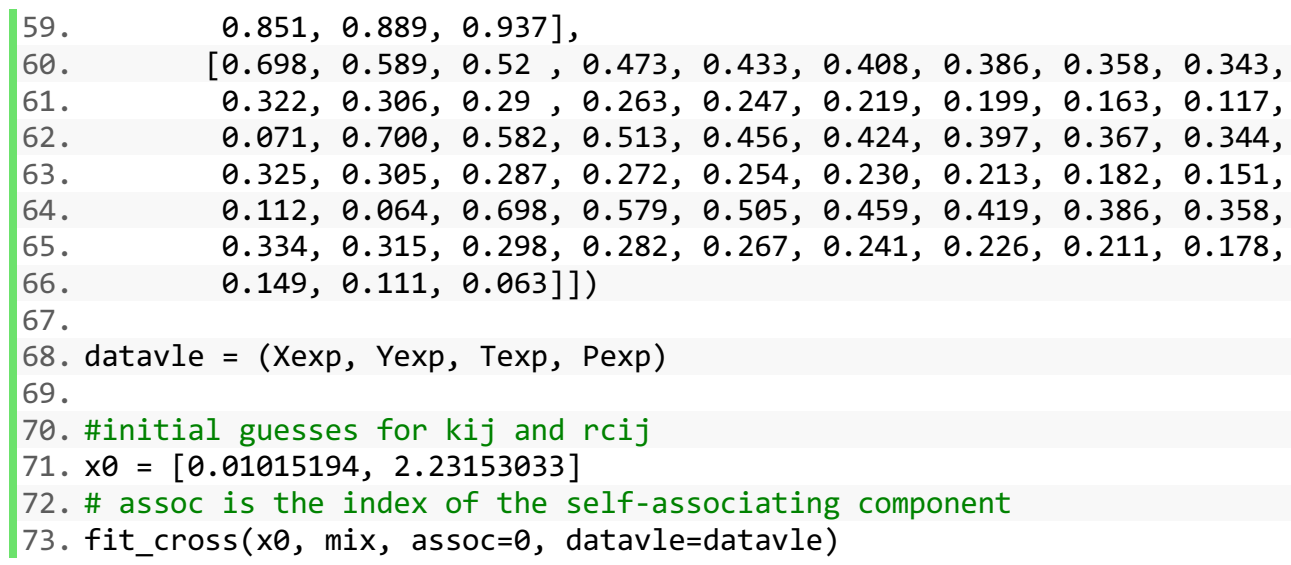

Notebook W15. Setup for the calculations for ethanol (1) + CPME (2) binary mixture.

The latter calculations illustrate the optimization of $k_{i j}$ and associating parameters. For the other binaries, the procedure is similar to the other examples already described. Once known all binary parameters, the first step to create a ternary mixture is to create the mixture and include the interaction parameters, as it shown below.

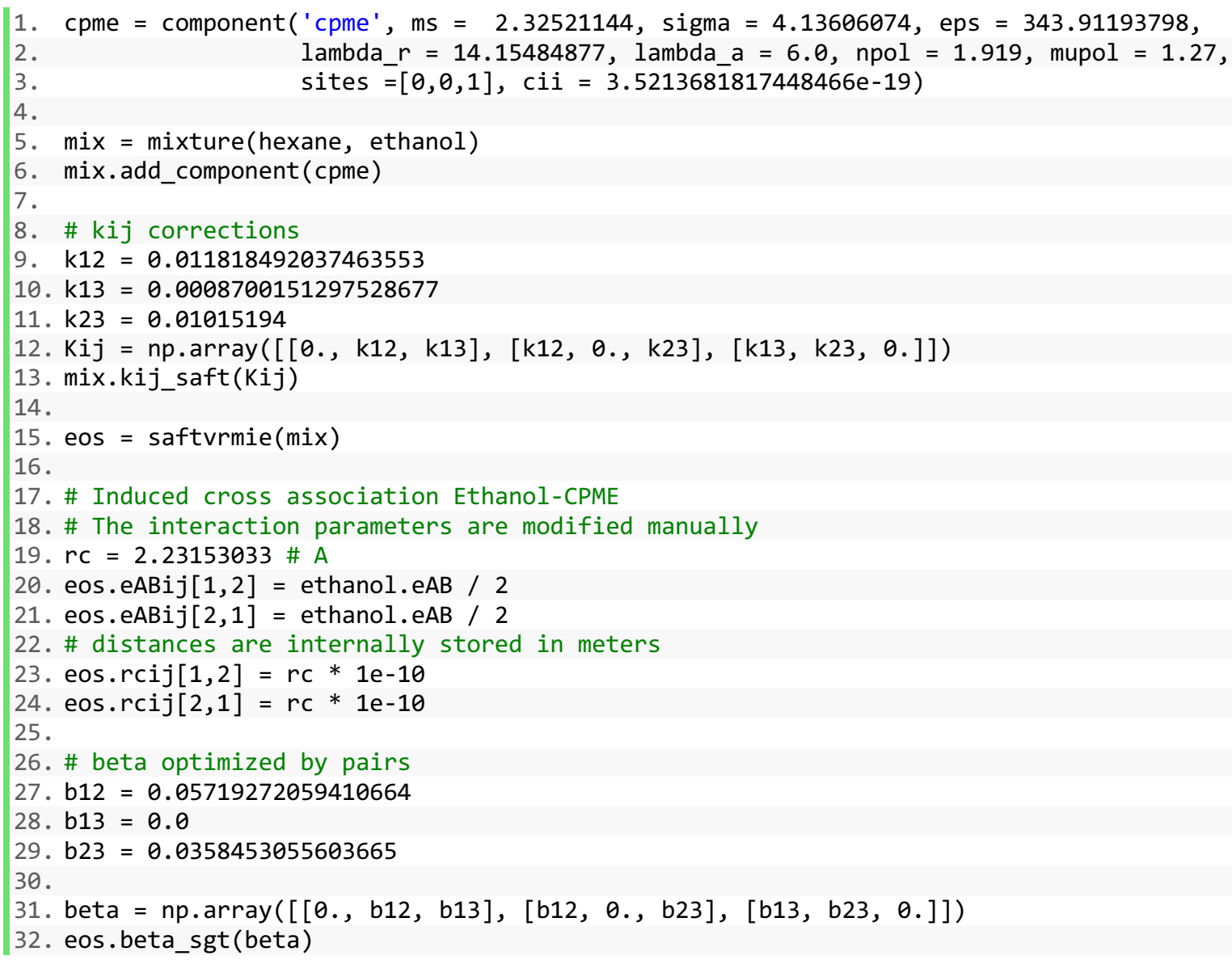


Notebook W16. Setup for the prediction of phase equilibrium and interfacial tensions for hexane (1) + ethanol (2) + CPME (3) ternary mixture.

In the previous notebook, we employ both $\beta_{i j}=0$ and $\beta_{i j} \neq 0$. The $\beta_{i j}$ values have been obtained from the binary subsystems. The numerical values and their deviation in interfacial tension $(\mathrm{AAD} \gamma)$ are $\beta_{12}=0.0572(\mathrm{AAD} \gamma=6.24 \%), \beta_{13}=0(\mathrm{AAD} \gamma=2.28 \%)$, and $\beta_{23}=0.036(\mathrm{AAD} \gamma=$ $4.44 \%)$. Following, the eos object can be used for phase equilibria and interfacial properties computation for the ternary mixture. To exemplify this process, a single point of the mixture is selected: $x_{1}=0.906$ and $x_{2}=0.71$ at $298.15 \mathrm{~K}$. Similar than binary mixtures, the first step is to compute the equilibrium pressure and vapor composition at the given conditions, then SGT is applied using the sgt_mix function. This procedure is shown below.

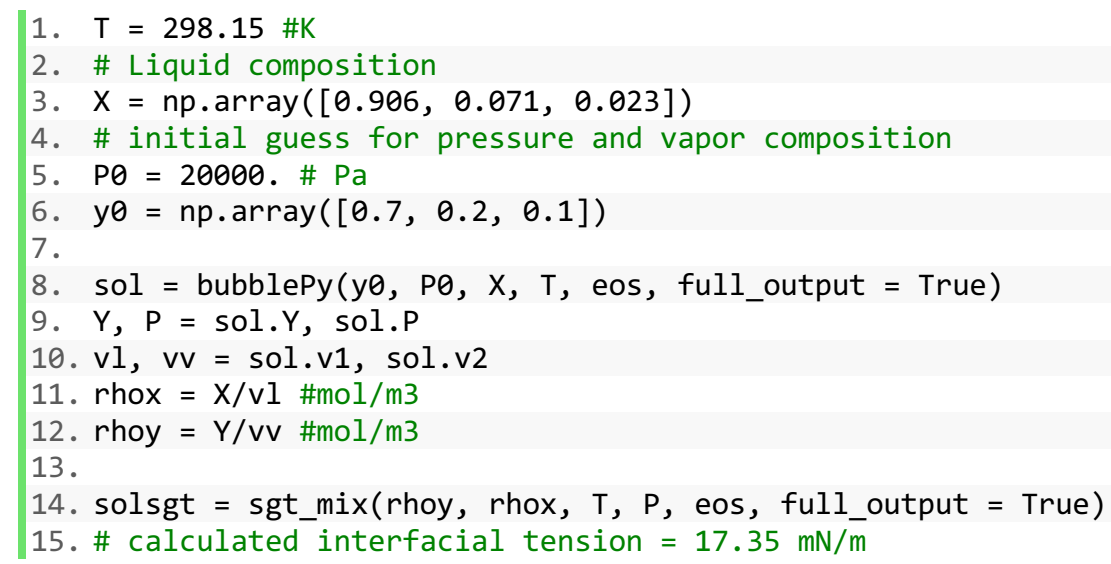

Notebook W17. Phase equilibrium and interfacial tensions for hexane (1) + ethanol (2) + CPME (3) ternary mixture at $x_{1}=0.906$ and $x_{2}=0.71$ at $298.15 \mathrm{~K}$.

From Notebook W17, the predicted interfacial tension is $17.35 \mathrm{mN} / \mathrm{m}$ and the experimental data at the given condition is $18.41 \mathrm{mN} / \mathrm{m} .{ }^{35}$ This procedure can be repeated at other compositions, allowing us to study the interfacial behavior of the mixture. Figure W6 displays the concentration profiles for hexane (1), ethanol (2), and CPME (3) at three equilibrium conditions. One appreciates that CPME behaves monotonically across the interface while ethanol and hexane accumulate at the interface, which is reflected by a positive surface activity. This latter behaviour decreases as the hydrocarbon concentration increases. 

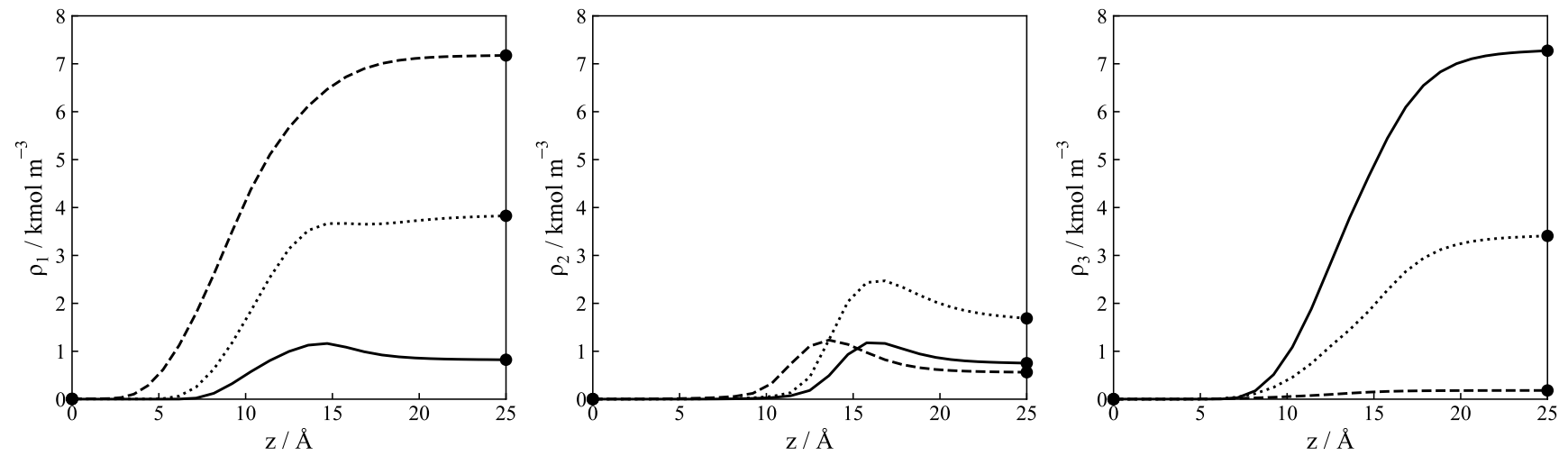

Figure W6. Concentration interfacial profile in $\rho_{i}-z$ projection for hexane (1) + ethanol (2) + CPME (3) mixture. ---, $x_{1}=0.906$ and $x_{2}=0.071 ;-, x_{1}=0.429$ and $x_{2}=0.189 ; \cdots, x_{1}=0.093$ and $x_{2}=0.085 ; \bullet$, bulk phase equilibrium.

Finally, the SGTPy is used to compute both isobaric phase equilibrium at $94 \mathrm{kPa}$ and isothermal interfacial tension at $298.15 \mathrm{~K}$ for this ternary mixture, and the results are compared to the available experimental data, ${ }^{35}$ as it is illustrated in Figure W7. Specifically, Figure W7.a compares the predicted phase equilibria results to the experimental data. The model predicts the phase equilibrium with very good agreement in terms of both mole fraction of vapor and temperature. In fact, the vapor phases are calculated with an absolute average deviation (AAD) of $\mathrm{AAD} y_{1}=0.22$ $\%$ and $\mathrm{AAD} y_{2}=0.64$, whereas for the bubble temperature, $\mathrm{AAD} T=0.07 \%$. Figure W7.b shows the predicted interfacial tension obtained with SGT is compared with the experimental data, the computed values predict the experiments within $3.18 \%$. 


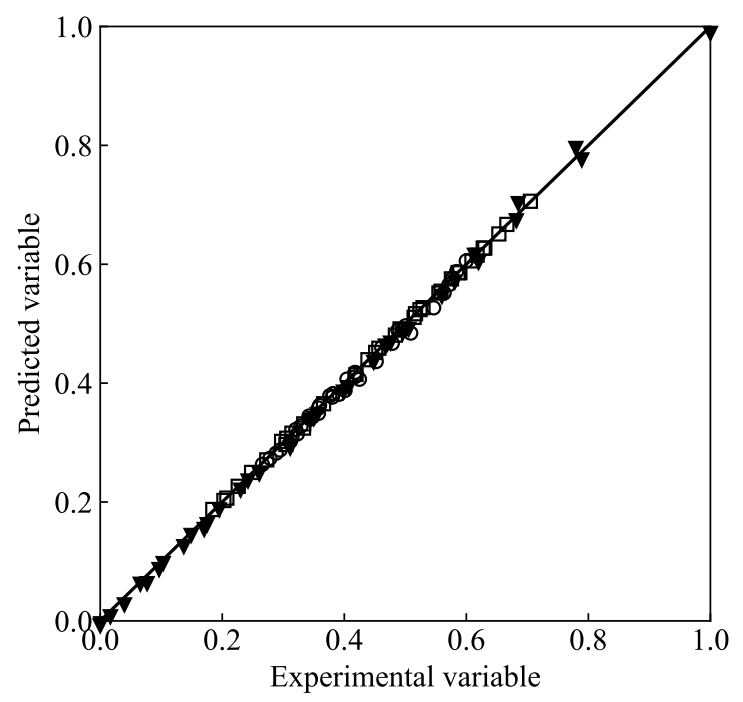

a. Phase Equilibrium at $94 \mathrm{kPa}$

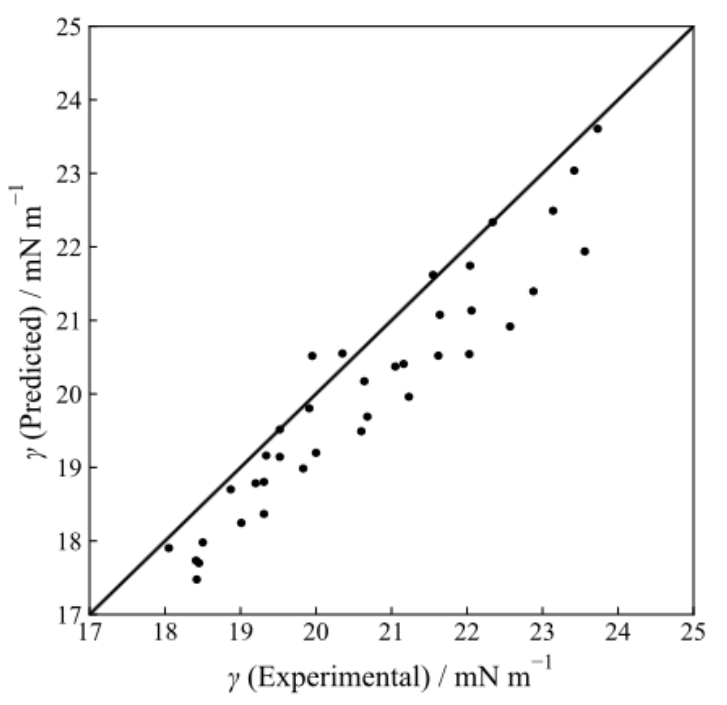

b. Interfacial tension at $298.15 \mathrm{~K}$

Figure W7. Predictions of phase equilibria an interfacial tension of hexane (1) + ethanol (2) + CPME (3) ternary mixture. $\boldsymbol{\nabla}$, reduced temperature $\left.\left(T^{*}=\left[\mathrm{T}-\min \left(\mathrm{T}^{\exp }\right)\right] /\left[\max \left(\mathrm{T}^{\exp }\right)-\min \left(\mathrm{T}^{\exp }\right)\right]\right)\right] ; \square$, vapor mole fraction for hexane $\left(y_{1}\right)$; $\bigcirc$, vapor mole fraction for ethanol $\left(y_{2}\right)$.

\section{Binary mixtures in liquid-liquid equilibria (LLE)}

In the previous examples, SGTPy was used to predict and correlate the interfacial behavior for binary and ternary mixtures in VLE using both $\beta_{i j}=0$ and $\beta_{i j} \neq 0$. Similar procedure can be applied to binary mixtures in liquid-liquid equilibria (LLE). In the following example, the mixture of water (1) + n-butanol (2) is considered. The first step, as we described before, is to define the pure components, the mixture, and its interaction parameters. In this mixture, it is necessary to optimize two mixture parameters, $k_{i j}$, and $l_{i j}$ as it is illustrated in the following notebook.

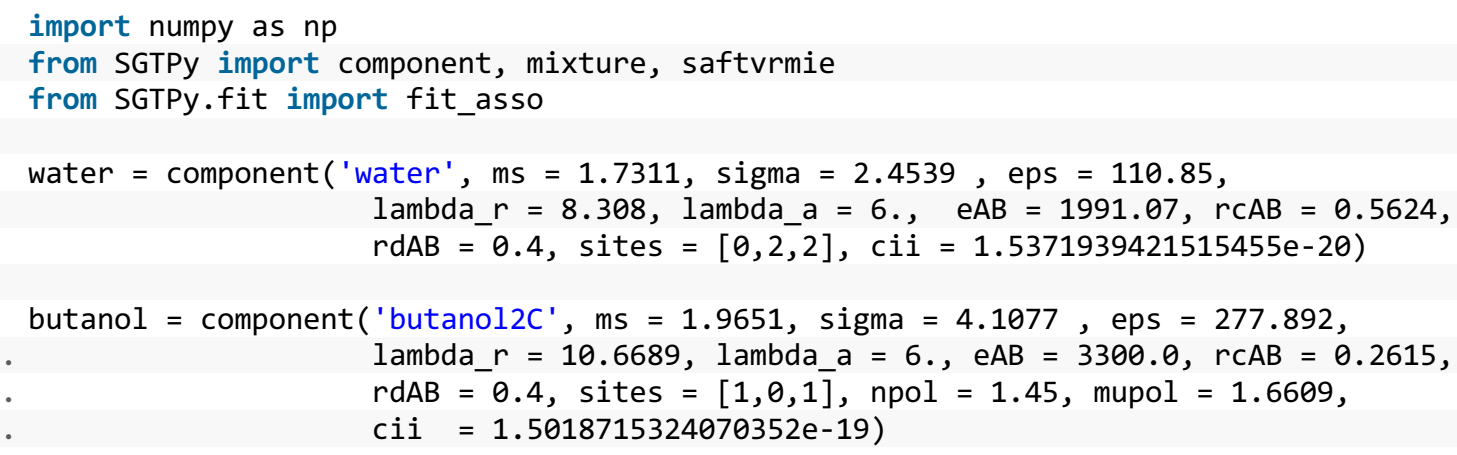




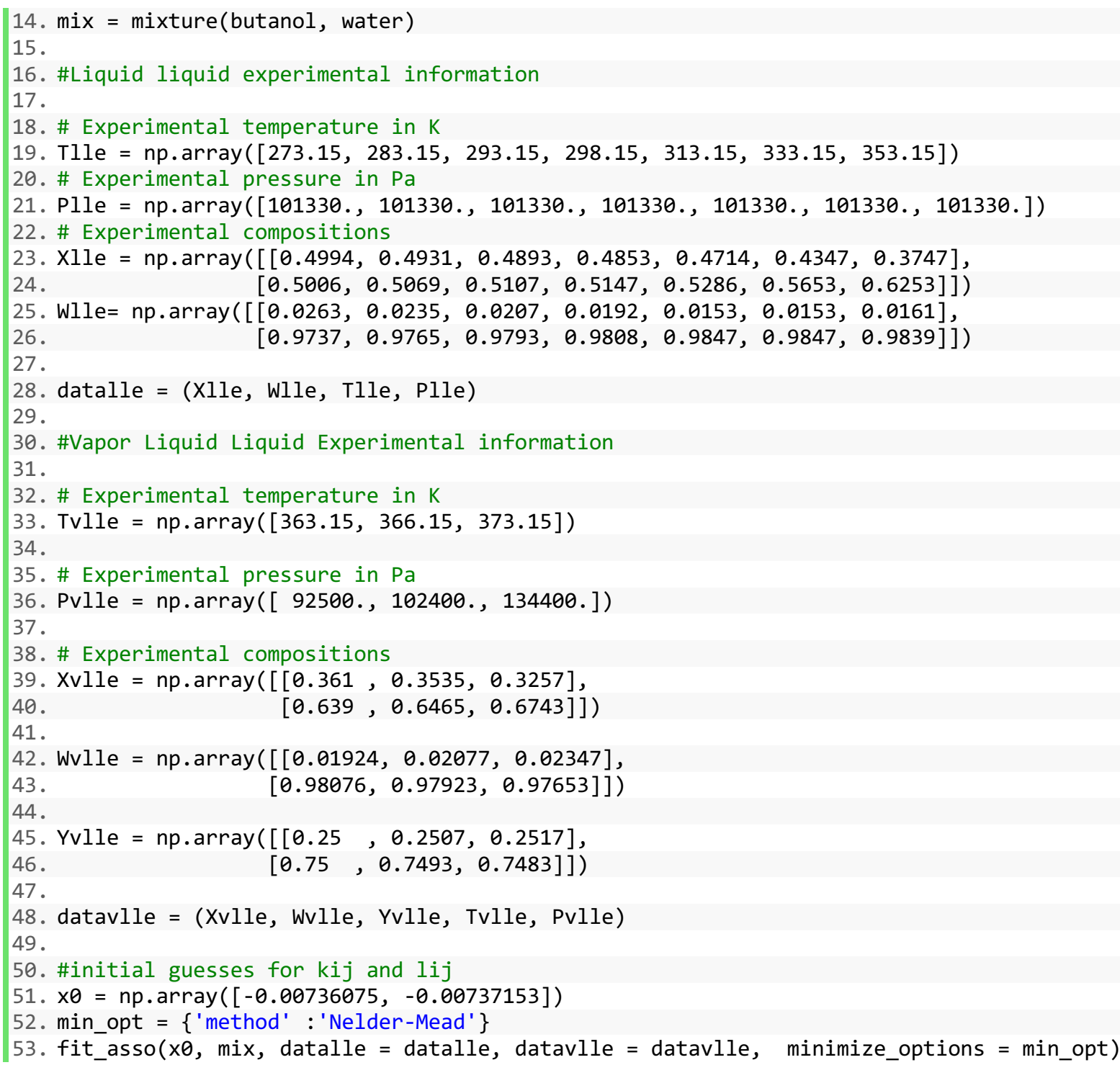

Notebook W18. Optimization of the binary parameter $k_{i j}$, and $l_{i j}$ for water (1) + n-butanol (2) mixture.

Once the pure and interaction parameters are known, a notebook is built as it is described below.

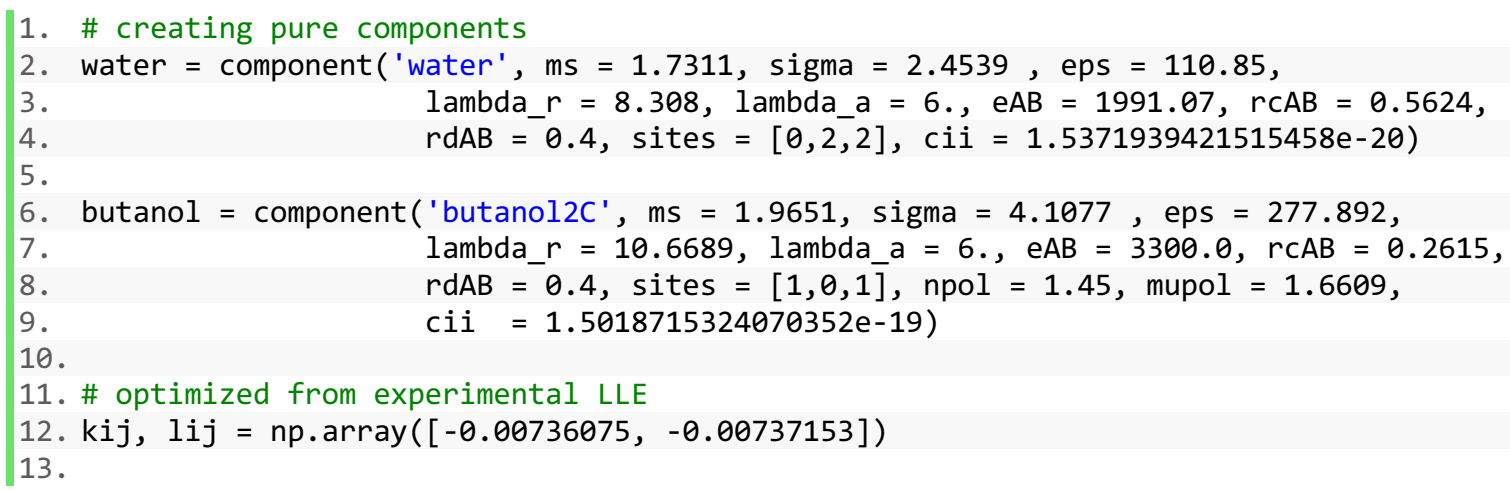




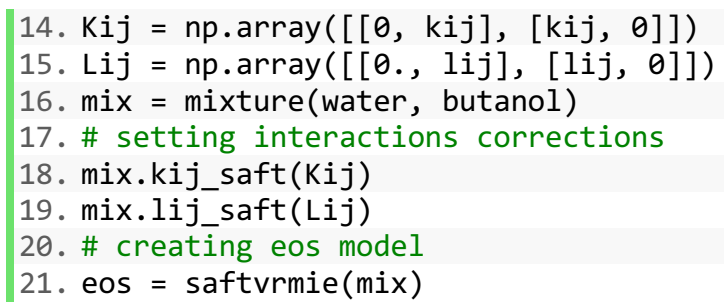

Notebook W19. Initial setup for the water (1) + n-butanol (2) mixture.

Once the eos object is available, it can be used to compute phase equilibria and interfacial properties using SGT. LLE is computed with lle function as a modified-flash strategy that verifies the stability of the phases. In order to ensure the convergence to the solution good initial compositions of the phases are generated by minimizing the tangent plane distance function with the lle_init function. Once the equilibrium results are available, the density vectors are computed and then SGT is applied, as illustrated in the following notebook.

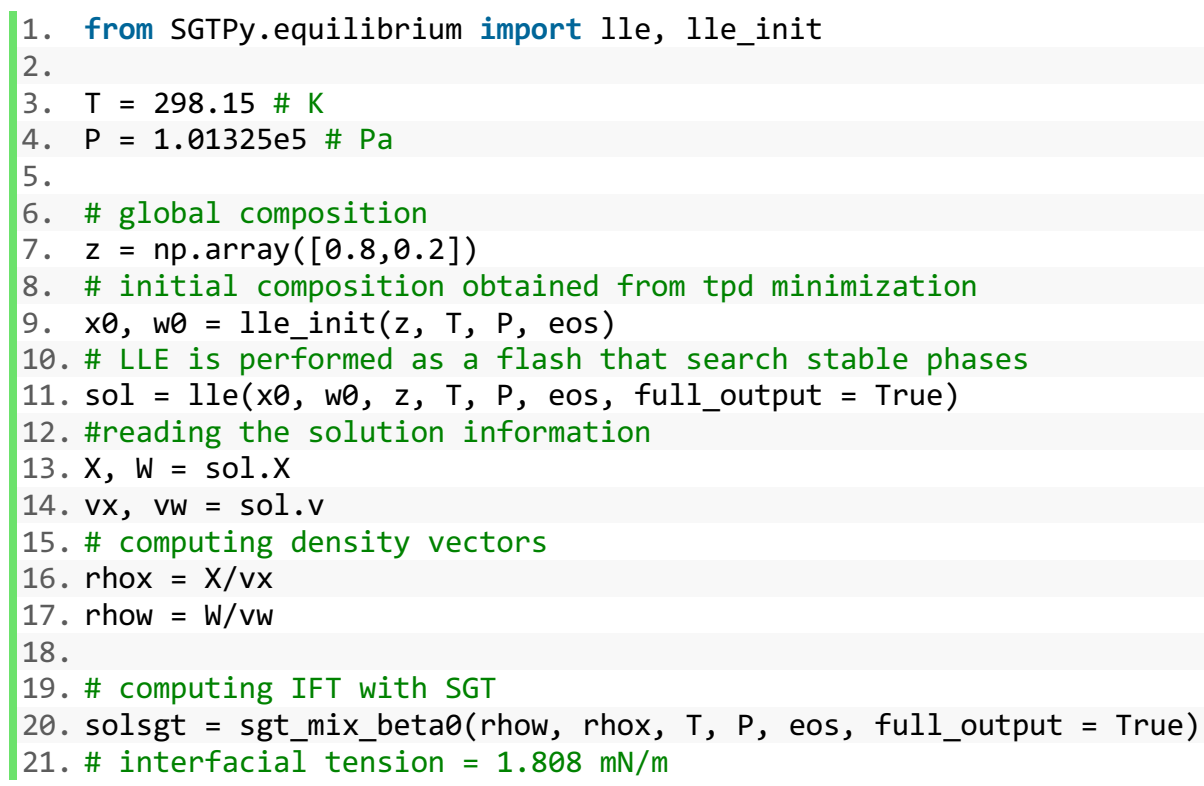

Notebook W20. Liquid - liquid and interfacial tension for water (1) + n-butanol (2) mixture.

From the last notebook, the computed interfacial of $1.808 \mathrm{mN} / \mathrm{m}$, which is close to the experimental value $1.68 \mathrm{mN} / \mathrm{m}$. For this equilibrium type, the density profiles usually behave monotonically through the interface, as it is shown in Figure W8.a. The same procedure is repeated over the whole temperature range to obtain the variation of the interfacial tension with temperature as it is illustrated in Figure W8.b. 


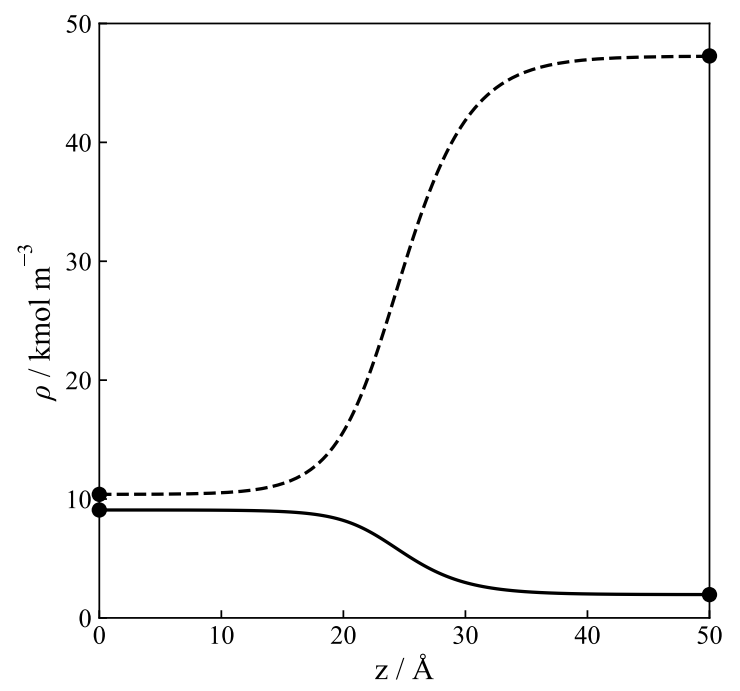

a. Concentration profile. ---, water; - , n-butanol; $\bullet$, bulk phase equilibrium at $298.15 \mathrm{~K}$

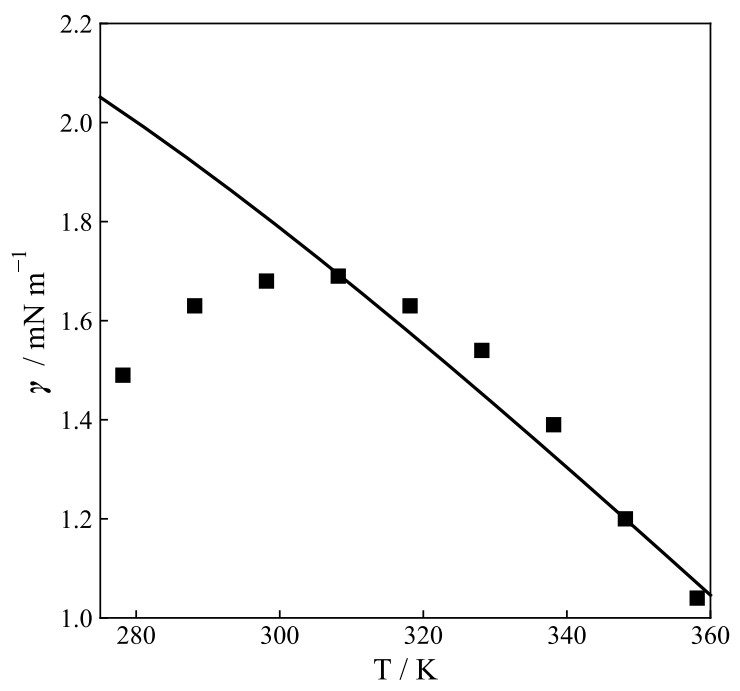

b. Interfacial tension as function of temperature

Figure W8. Interfacial properties for water + n-butanol mixture at LLE at $101.3 \mathrm{kPa}$. - , predicted; Cardenas et al., ${ }^{36}$

In Figure W8.b the non-monotonic behaviour of the tension is observed, presenting a maximum with temperature, a feature that the SGT fails to capture. This disparity is related to the inability of the EoS model to accurately describe the bulk phase density with a single $k_{i j}$ parameter and the values of the influence parameters were obtained from vapor-liquid equilibrium. In order to obtain a better agreement between experimental data and theoretical predictions, other values of $\beta$ were explored but $\beta=0$ reports the minimum deviation. As was described by Danzer and Enders ${ }^{37}$ and Haarmann et al., ${ }^{38}$ some improvement can be obtained by using a second-order dependence of the influence parameters as well as cross interaction parameters. However, the application of SGT for LLE needs further refinements, as well as theoretical improvements. 


\section{Ternary mixture in vapor-liquid-liquid equilibria (VLLE)}

A final illustration of the capabilities and options of SGTPy to describe the interfacial properties of mixtures is the case of interfacial properties of mixtures in VLLE. As it was described in section 4 , both phase equilibrium and their interfacial tension (i.e., $\gamma^{V L 1}, \gamma^{V L 2}$, and $\gamma^{L L L 2}$ ) were calculated for the water (1) + n-butanol (2) + MTBE (3) ternary mixture. The following Notebook illustrates this case.

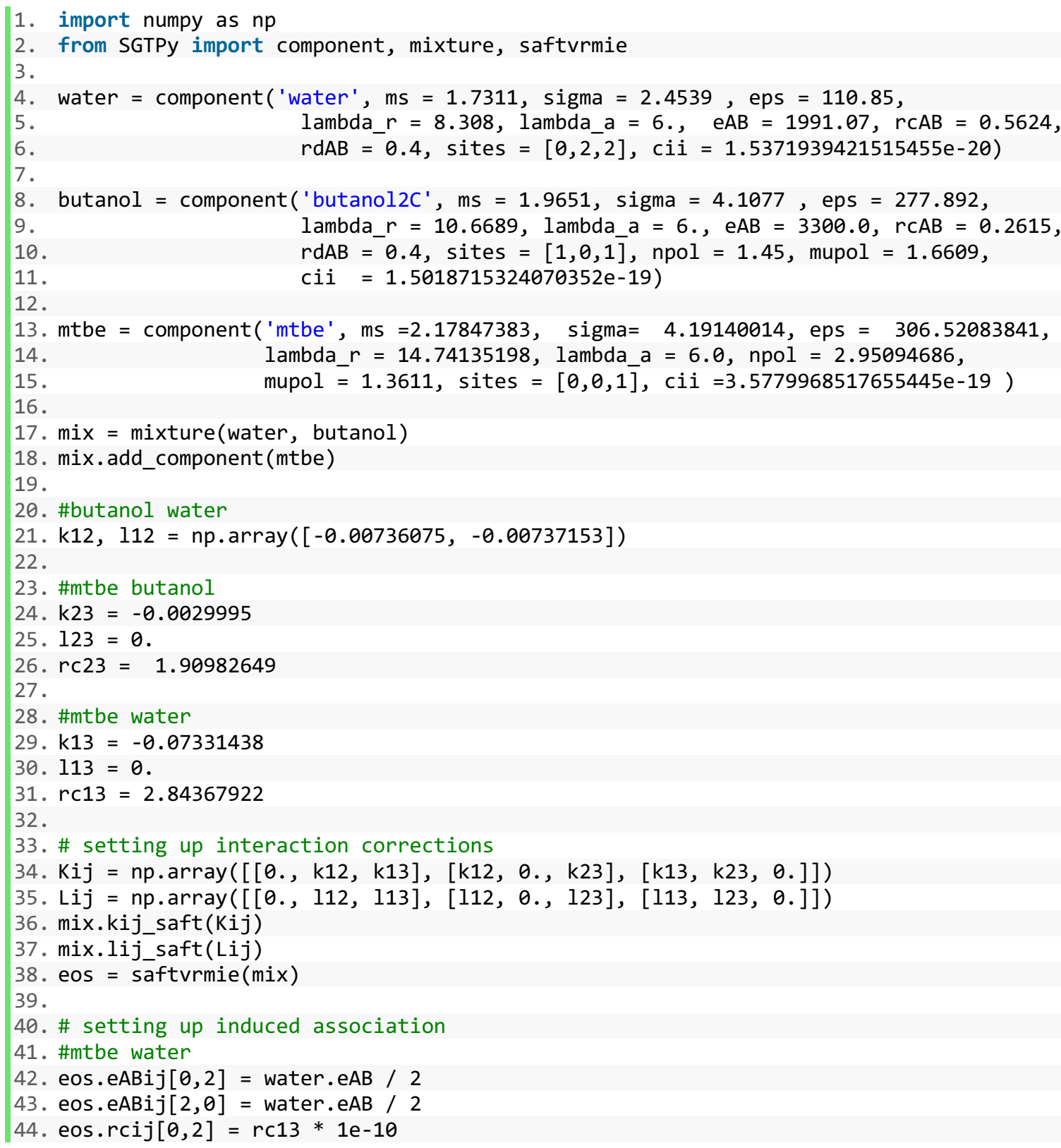




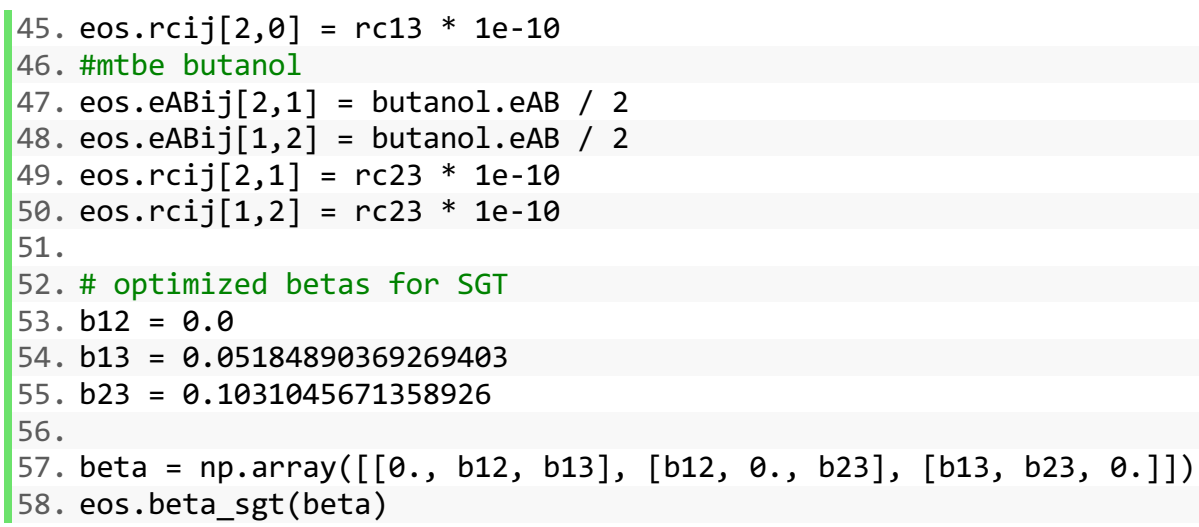

Notebook W21. Setup for phase equilibrium and interfacial properties for water (1) + n-butanol (2) + MTBE (3) mixture.

Once the phase equilibrium is obtained, the density vector is computed and SGT applied, as it is shown in the following code-block.

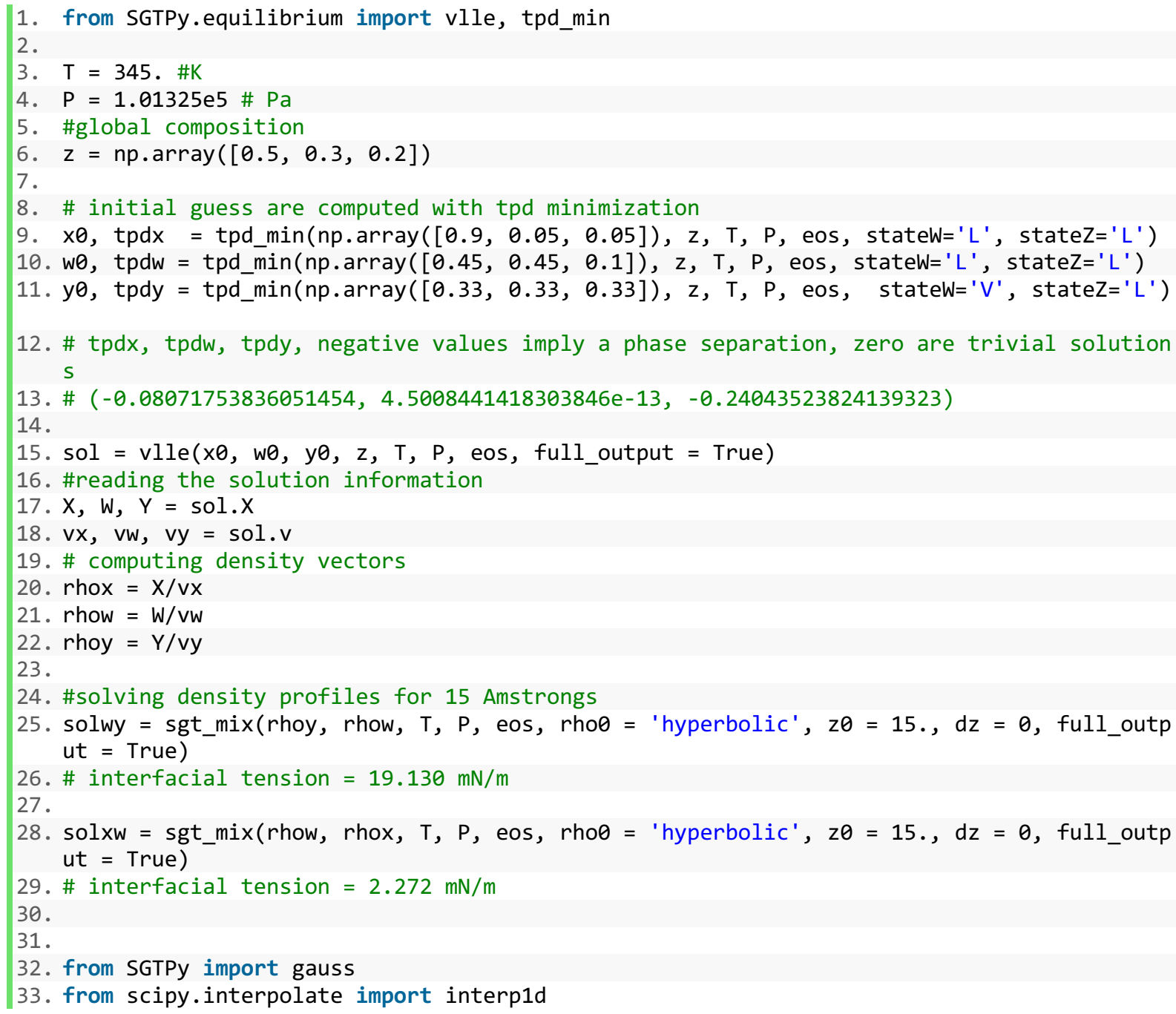




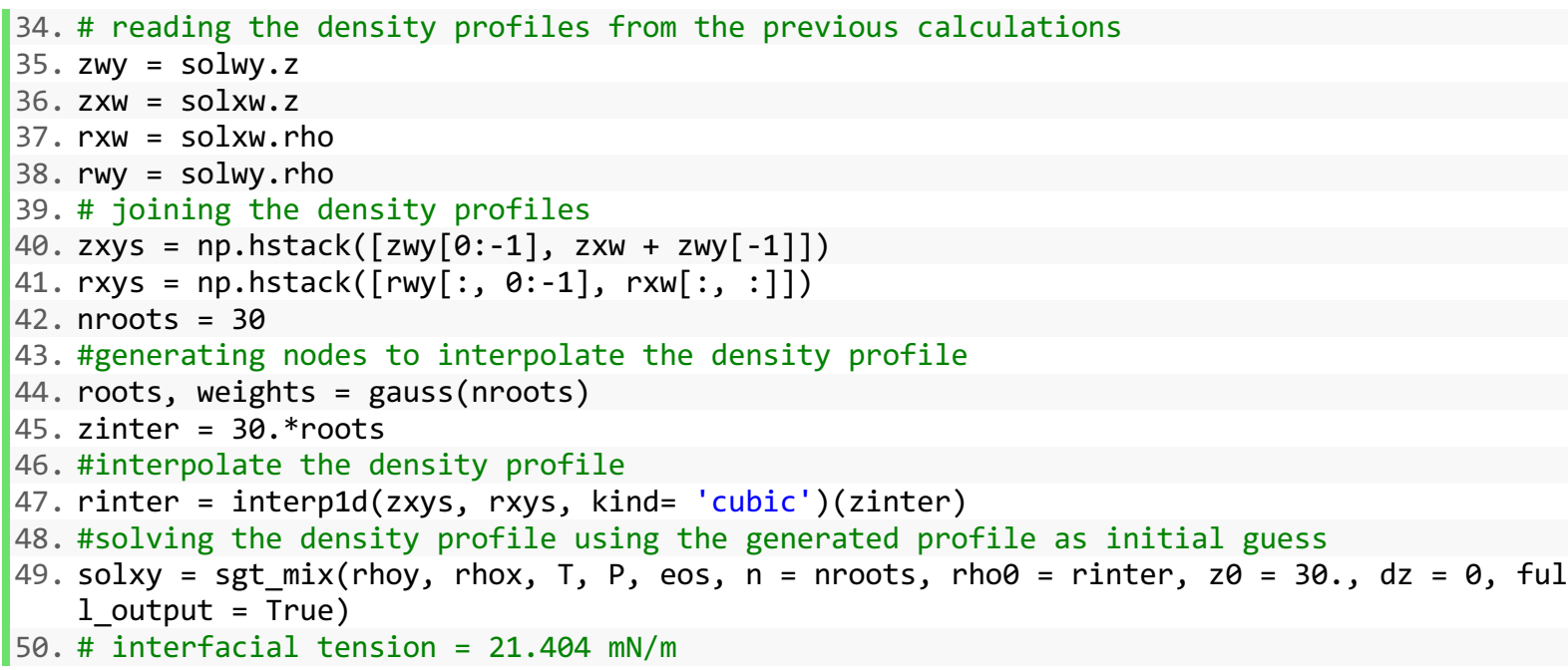

Notebook W22. Phase equilibrium and interfacial properties for water (1) + n-butanol (2) + MTBE (3) mixture at $345 \mathrm{~K}$ and $101.3 \mathrm{kPa}$.

It is important to point out that SGTPy solves the BVP, Equation (1), starting with linear or hyperbolic-tangent-like density profiles as initial guesses. These starting profiles work fine for most of the cases, as for the interfaces of the organic-vapor and organic-aqueous phases shown in previous examples. In some cases, as for the aqueous-vapor interface of this ternary three-phase equilibria, a better initial profile is required. For this latter case, an alternative procedure is carried out. The initial profile is formed by joining the previously computed density profiles and interpolating them at the desired grid.

\section{Mixtures with unknown behavior}

The illustrated examples are based on well known systems where the phase behavior is known beforehand although SGTPy can be also used to explore systems of unknown behavior. In this scenario, a mixture at given global composition, temperature and pressure is considered. The first step is to check the stability of the mixture using the tangent plane distance $(t p d)$. The tpd function is minimized, and all minima are analysed. In the case where all minima are found positive, the mixture is stable and no phase split will occur. In the case of any minimum is negative, the mixture will split into a VLE, LLE or VLLE (depending on the number of minima found and their aggregation state). In any of those cases, the phase equilibria and the interfacial behaviour can be computed with SGTPy as it is illustrated in Figure W9. 


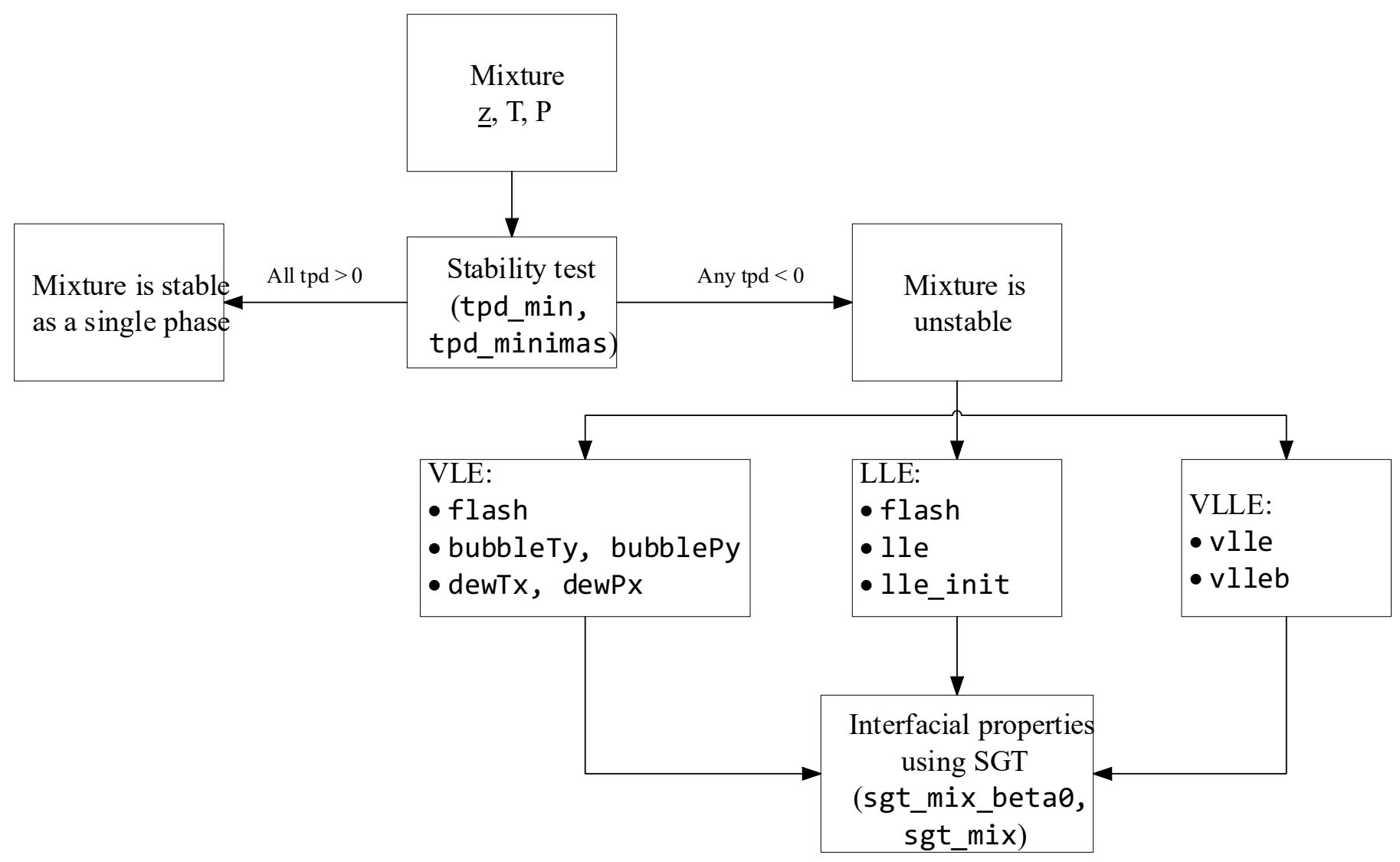

Figure W9. Illustration of the SGTPy for exploring mixtures of unknown behavior.

The SGTPy code as well as the notebooks used for the illustrations described here can be loaded, free of charge, from https://github.com/gustavochm/SGTPy.

\section{Acknowledgment}

This work was financed by FONDECYT, Santiago, Chile (Project. 1190107). E.A.M. further acknowledges the support from EPSRC through research grants to the Molecular Systems Engineering group (grant nos. EP/E016340, EP/J014958, and EP/R013152) 


\section{References}

(1) Chaparro, G.; Mejía. A. Phasepy: a Python based framework for fluid phase equilibria and interfacial properties computation. J. Comp. Chem. 2020, 41, 2504-2526

(2) van der Walt, S.; Colbert, S. C.; Varoquaux, G. The NumPy array: A structure for efficient numerical computation. Comput. Sci. Eng. 2011, 13, 22-30.

(3) Virtanen, P.; Gommers, R.; Oliphant, T. E.; Haberland, M.; Reddy, T.; Cournapeau, D.; Burovski, E.; Peterson. P.; Weckesser, W.; Bright, J.; van der Walt, J.; Brett, M.; Wilson, J.; Millman, K. J.; Mayorov, M.; Nelson, A. R. J.; Jones, E.; Kern, R.; Larson, E.; Carey, C.J.; Polat, İ.; Feng, Y.; Moore, E. W.; van der Plas, J.; Laxalde, D.; Perktold, J.; Cimrman, R.; Henriksen, I.; Quintero, E. A.; Harris, C. R.; Archibald, A. M.; Ribeiro, A. H.; Pedregosa, F.; van Mulbregt, P. SciPy 1.0: fundamental algorithms for scientific computing in Python. Nat. Methods. 2020, 17, 261-272.

(4) Behnel, S.; Bradshaw, R.; Citro C.; Dalcin, L.; Seljebotn, D. S.; Smith, K. Cython: The best of both worlds. Comput. Sci. Eng. 2011, 13, 31-39.

(5) Python Software Foundation, Python (2020), Accessed on 2020-11-02, https://www.python.org/

(6) Python Software Foundation, PyPI (2020), Accessed on 2020-11-02, https://pypi.org/

(7) Tan, S. P.; Adidharma, H.; Radosz, M. Generalized procedure for estimating the fractions of nonbonded associating molecules and their derivatives in thermodynamic perturbation theory. Ind. Eng. Chem. Res. 2004, 43, 203-208.

(8) Michelsen, M. L. Robust and efficient solution procedures for association models. Ind. Eng. Chem. Res. 2006, 45, 8449-8453.

(9) Cripwell, J. T.; Smith, S. A. M.; Schwarz, C. E.; Burger, A. J. SAFT-VR Mie: Application to phase equilibria of alcohols in mixtures with n-alkanes and water. Ind. Eng. Chem. Res. 2018, 57, 9693-9706.

(10) Dufal, S.; Lafitte, T.; Haslam, A. J.; Galindo, A.; Clark, G. N. I.; Vega, C.; Jackson, G. The A in SAFT: developing the contribution of association to the Helmholtz free energy within a Wertheim TPT1 treatment of generic Mie fluids. Mol. Phys. 2015, 113, 948-984.

(11) DECHEMA Gesellschaft für Chemische Technik und Biotechnologie e.V., Frankfurt am Main, Germany, https://i-systems. dechema.de/detherm/mixture.php/ (last visited 02/11/2020). 
(12) Diky, V.; Chirico, R. D.; Frenkel, M.; Bazyleva, A.; Magee, J. W.; Paulechka, E.; Kazakov, A.; Lemmon, E. W.; Muzny, C. D.; Smolyanitsky, A. Y.; Townsend, S.; Kroenlein, K. ThermoData Engine (TDE) version 10.1 (Pure Compounds, Binary Mixtures, Ternary Mixtures and Chemical Reactions), NIST Standard Reference Database \# 103b. Thermodynamics Research Center (TRC), Applied Chemicals and Material Division. Standard Reference Data Program; National Institute of Standards and Technology (NIST) 2016.

(13) Mejía, A; Herdes, C.; Müller, E. A. Force fields for coarse-grained molecular simulations from a corresponding states correlation. Ind. Eng. Chem. Res. 2014, 53, 4131-4141.

(14) Mejía. A.; Cartes, M. Experimental determination of isobaric vapor-liquid equilibrium and isothermal interfacial tensions for the binary ethanol + cyclopentyl methyl ether mixture. J. Chem. Eng. Data, 2019, 64, 1970-1977.

(15) Topliss, R. J.; Dimitrelis, D.; Prausnitz, J. M. Computational aspects of a non-cubic equation of state for phase-equilibrium calculations. Effect of density-dependent mixing rules. Comput. Chem. Eng. 1988, 12, 483-489.

(16) Garrido, J. M.; Piñeiro, M. M.; Blas, F. J.; Müller, E. A.; Mejía, A. Interfacial tensions of industrial fluids from a molecular-based square gradient theory. AIChE J. 2016, 62, 1781-1794.

(17) Srivastan, S.; Darwish, N. A.; Gasem, K. A. M.; Robinson, R. L. Solubility of methane in hexane, decane, and dodecane at temperatures from 311 to $423 \mathrm{~K}$ and pressures to $10.4 \mathrm{MPa}$. J. Chem. Eng. Data. 1992, 37, 516-520.

(18) Sinor J. E.; Weber, J. H. Vapor-liquid equilibria at atmospheric pressure systems containing ethyl alcohol, n-hexane, benzene and methylcydopentane. J. Chem. Eng. Data, 1960, 5, 243-247. (19) Mejía, A; Cartes, A. Experimental determination of isobaric vapor-liquid equilibrium and isothermal interfacial tensions for the binary ethanol + cyclopentyl methyl ether mixture. J. Chem. Eng. Data. 2019, 64, 1970-1977.

(20) Mejía. A.; Cartes, M.; Chaparro, G. Isobaric vapor-liquid equilibrium and isothermal surface tension for hexane + cyclopentyl methyl ether binary mixture: Experimental determinations and theoretical predictions. Fluid Phase Equilib. 2020, 520, 1-10.

(21) Arce, A.; Rodil, E.; Soto, A. Extractive distillation of 2-methoxy-2-methylpropane + ethanol using 1-butanol as entrainer: Equilibria and simulation. Can. J. Chem. Eng. 1999, 77, 1135-1140. (22) Marongiu, B.; Ferino, I.; Monaci, R.; Solinas, V.; Torrazza, S. Thermodynamic properties of aqueos non-electrolyte mixtures. Alkanols + water systems. J. Mol. Liq. 1984, 28, 229-247. 
(23) Lladosa, E.; Montón, J. B.; Cruz Burguet, M.; Muñoz, R. Phase equilibrium for the esterification reaction of acetic acid + butan-1-ol at $101.3 \mathrm{kPa}$. J. Chem. Eng. Data. 2008, 53, 108115.

(24) Zikmundová, D.; Matouš, J.; Novák, J. P.; Kubíček, V.; Pick, J. Liquid-liquid and vapourliquid equilibria in the system methyl tert-butyl ether + tetrahydrofuran + water. Fluid Phase Equilib. 1990, 54, 93-110.

(25) Ashour, I. Liquid-liquid equilibrium of MTBE + ethanol + water and MTBE + 1-hexanol + water over the temperature range of 288.15 to 308.15 K. J. Chem. Eng. Data. 2005, 50, 113-118. (26) Stephenson, R.; Stuart, J. Mutual binary solubilities: water-alcohols and water-esters. J. Chem. Eng. Data. 1986, 31, 56-70.

(27) Cartes, M. Private comunication, 2020.

(28) Mejía, A.; Cartes, M.; Segura, H.; Müller, E. A. Use of equations of state and coarse grained simulations to complement experiments: describing the interfacial properties of carbon dioxide + decane and carbon dioxide + eicosane mixtures, J. Chem. Eng. Data, 2014, 59, 2928-2941.

(29) Cumicheo, C.; Cartes, M.; Segura, H.; Müller, E. A.; Mejía, A. High-pressure densities and interfacial tensions of binary systems containing carbon dioxide + n-alkanes: (n-dodecane, $n$ tridecane, n-tetradecane). Fluid Phase Equilib. 2014, 380, 82-92.

(30) Cumicheo, C.; Cartes, M.; Müller, E. A; Mejía, A. Experimental measurements and theoretical modeling of high-pressure mass densities and interfacial tensions of carbon dioxide + n-heptane + toluene and its carbon dioxide binary systems. Fuel. 2018, 228, 92-102.

(31) Liang, T. X.; Michelsen, M. L.; Kontogeorgis, G. M. Pitfalls of using the geometric-mean combining rule in the density gradient theory. Fluid Phase Equilib. 2016, 415, 75-83.

(32) Liang, T. X.; Michelsen, General approach for solving the density gradient theory in the interfacial tension calculations. Fluid Phase Equilib. 2017, 451, 79-90.

(33) Jiménez, E.; Casas, H.; Segade, L.; Franjo, C. Surface tensions, refractive indexes and excess molar volumes of hexane+1-alkanol mixtures at 298.15 K. J. Chem. Eng. Data. 2000, 45, 862866.

(34) Cornelisse, P. M. W. The squared gradient theory applied. Simultaneous modelling of interfacial tension and phase behaviour. Ph.D. thesis, Delft University. 1997.

(35) Chaparro, G.; Cartes, M.; Mejía, A. Vapor-liquid equilibrium at $94 \mathrm{kPa}$ and surface tension at $298.15 \mathrm{~K}$ for hexane + ethanol + cyclopentyl methyl ether mixture. Fuel. 2020, 279, 118415. 
(36) Cárdenas, H.; Cartes, M.; Mejía, A. Atmospheric densities and interfacial tensions for 1alkanol (1-butanol to 1-octanol) + water and ether (MTBE, ETBE, DIPE, TAME and THP) + water demixed mixtures, Fluid Phase Equilib. 2015, 396, 88-97.

(37) Danzer, A. Enders, S. Comparison of two modelling approaches for the interfacial tension of binary aqueous mixtures. J. Mol. Liq. 2018, 266, 309-320.

(38) Haarmann, N.; Reinhardt, A.; Danzer, A.; Sadowski, G. Enders, S. Modeling of interfacial tensions of long-chain molecules and related mixtures using perturbed chain-statistical associating fluid theory and the density gradient theory. J. Chem. Eng. Data. 2020, 65, 1005-1018. 